\author{
RESEARCH ARTICLE \\ 10.1029/2018JC014747 \\ This article is a companion to \\ Kudryavtsev et al. (2019), https://doi. \\ org/10.1029/2018JC014746. \\ Key Points: \\ - Simplified analytical model for \\ baroclinic and barotropic ocean \\ response to $\mathrm{TC}$ and its impact on \\ pycnocline erosions is suggested \\ - The model demonstrated its ability \\ to reproduce $\mathrm{SSH}$ and SST \\ observations in wide range of TC \\ parameters and ocean stratification \\ - Given its low computational burden, \\ the model can be introduced as a \\ computational module into \\ numerical coupled TC-ocean models
}

Correspondence to:

V. Kudryavtsev,

kudr@rshu.ru

Citation:

Kudryavtsev, V., Monzikova, A., Combot, C., Chapron, B., \& Reul, N. (2019). A simplified model for the baroclinic and barotropic ocean response to moving tropical cyclones: 2 . Model and simulations. Journal of Geophysical Research: Oceans, 124, 3462-3485. https://doi.org/10.1029/ 2018JC014747

Received 7 NOV 2018 Accepted 28 MAR 2019 Accepted article online 26 APR 2019 Published online 31 MAY 2019

(c)2019. American Geophysical Union. All Rights Reserved.

\section{A Simplified Model for the Baroclinic and Barotropic Ocean Response to Moving Tropical Cyclones: 2. Model and Simulations}

\author{
Vladimir Kudryavtsev ${ }^{1,2}$ (D), Anna Monzikova ${ }^{1}$, Clément Combot ${ }^{3}$, Bertrand Chapron ${ }^{1,3}$, and \\ Nicolas Reul ${ }^{3}$ \\ ${ }^{1}$ Satellite Oceanography Laboratory, Russian State Hydrometeorological University, Saint-Petersburg, Russia, ${ }^{2}$ Remote \\ Sensing Department, Marine Hydrophysical Institute, Sevastopol, Russia, ${ }^{3}$ Laboratoire d'Oceanographie Physique et \\ Spatiale, Institut Français de Recherche pour l'Exploitation de la Mer, Plouzané, France
}

Abstract A simplified analytical model is developed to describe the baroclinic and barotropic ocean response to moving tropical cyclones (TCs) and their associated pycnocline erosions. The model builds on classical mixed-layer (ML) models and linear models of ocean response to transient events. As suggested, disturbances of the upper ocean stratification caused by the ML development shall not strongly impact the dynamics of baroclinic modes. Accordingly, the baroclinic response can be estimated using the prestorm ocean stratification condition. To the contrary, the ML is strongly coupled with these interior motions, through the TC-induced upwelling response that affects the entrainment velocity. The ML temperature is then strongly dependent on the local temperature gradient in the upper layer. The model is represented by a set of analytical relationships providing rapid calculations for the ocean response to TC, given a prescribed wind velocity field traveling over an ocean with arbitrary stratification. Compared to satellite observations, simulations demonstrate the model ability to quantitatively reproduce the observed shape and magnitudes of the sea surface height and the sea surface temperature (SST) anomalies. Remarkably, the model is robust and efficient for a wide range of variability of TC characteristics (max wind speed, radius, shape of wind profile, and translation velocity), parameters of the ocean stratification, and Coriolis parameter. Simulations provide solid evidences about the key role of TC-induced upwelling in the ML cooling and formation of SST wake. Cross-track advection by wind-driven currents, though small compared with TC translation velocity, can significantly contribute to broaden the shape and offset of the SST wake. Given its effectiveness and low computational burden, the proposed model can be introduced as a computational module into atmospheric numerical models of TC-coupled evolution with the ocean, through the resulting local changes of surface enthalpy fluxes.

\section{Introduction}

A complete description of upper ocean responses to tropical cyclone (TC) transient and extreme wind forcing remains a difficult problem. Crucial to quantify both the momentum increase of surface currents and the efficiency of vertical mixing in cooling the ocean surface, the parameterization of the wind forcing under extreme conditions is indeed still poorly known. Moreover, besides the combining effects of the TC intensity and translation speed, upper ocean precyclone stratification can also strongly mitigate or exacerbate TCinduced cooling amplitude (Lloyd \& Vecchi, 2011; Schade, 2000). The intensity-wake relationship can then be strongly modulated with evolving air-sea fluxes associated to sea surface temperature (SST) changes over the TC path (Cione \& Uhlhorn, 2003). Passage over freshwater plumes has also been reported to cause strengthening of hurricanes, due to the presence of a barrier layer effect (e.g., Balaguru et al., 2012; Reul et al., 2014).

To assess the sensitivity of the parameterization of wind forcing at high winds and/or the impact of precyclone upper ocean stratification (Yablonsky \& Ginis, 2013; Zedler et al., 2009; Zedler et al., 2012), advanced numerical ocean models are generally implemented to simulate the ocean response. These threedimensional and time-dependent models (e.g., Price et al., 1994; Sanford et al., 2007) consistently solve the momentum, heat, and salt budget equations. Numerical outputs then provide detailed descriptions of ocean response to moving TC, including description of wind-driven current field, ML cooling, its evolution, and the space-time variability of the pycnocline caused by TC-induced baroclinic motions. However, these 
advanced models require significant computer resources and simulations are time-consuming. This may appear as major hurdles to develop fully coupled ocean-atmosphere models and/or try to best sample the whole uncertainty space to apply inverse approaches, and more particularly to test and infer wind forcing parameterization.

In the latter context, different methods have been proposed. Sraj et al. (2013) proposed polynomial chaos expansions to construct a faithful surrogate of the response of upper ocean model simulations. For the same purpose, Zedler et al. (2013) adopt a numerical approach in an inverse problem setup, using the ocean model and its adjoint. Data can thus be assimilated and the drag coefficient adjusted, to help correspond to the minimum of a model minus data misfit or cost function.

To possibly serve these inverse approaches, as well as to derive a simplified framework to rapidly interpret satellite observations (e.g., Kudryavtsev et al., companion paper), it is tempting to circumvent the computational burden of advanced numerical simulations. This is the main purpose of the present paper. The goal is to develop a simplified analytical model to assess, on quantitatively correct levels, ocean responses to moving TCs.

As proposed, model derivations can essentially build on classical 1D mixed-layer models (e.g., Niiler, 1975; Zilitinkevich et al., 1979) and previously suggested analytical models for the barotropic and baroclinic responses (e.g., Geisler, 1970; Orlanski \& Polinsky, 1983). To rapidly outline the proposed developments, it is recalled that wind-driven currents, generated by a TC wind stress field, are localized in the mixed layer (ML). In the open ocean, the ML depth, $h$, is typically much smaller than the depth, $H$, of the quiet interior layer, $h<<H$. The ML layer is highly turbulent. The interior layer is not. Consequently, the TC-induced deepening of the ML shall lead to marked discontinuities in density and current velocity profile at the base of the ML, separating the highly turbulent upper ocean from the nonturbulent interior layer (Price, 1981). Caused by the vorticity of the TC surface wind stress, wind-driven currents in the ML are divergent, further leading to lift the interior layer, triggering inertia-gravity internal waves and associated baroclinic wake (e.g., Gill, 1982; Ginis, 2002). A barotropic response of the ocean to the wind stress vorticity action is also expected. Ginis and Sutyrin (1995) argued that barotropic and baroclinic modes do not interact and can thus independently be considered. Finally, feedbacks between ML and baroclinic motions can also encompass the impact of the thermocline upwelling on the ML cooling. Indeed, both numerical simulations and experimental observations (Yablonsky \& Ginis, 2009) provide solid evidences about the key role of TC-induced upwelling to control the ML cooling, especially for slowly translating TCs.

The paper is organized as the following. In section 6 we introduce the governing equations for the upper ML (sections 6.1 and 2.2) and the interior layer (section 6.3). Simplification of the governing equations and analytical solutions describing baroclinic and barotropic responses to moving TC, and the SST anomalies is given in section 3. Section 4 presents some results of model simulations, demonstrating general properties of the model. Model simulations are applied to compare with satellite observations of the SST and the surface height anomalies, reported in the companion paper, and are presented in section 5. Conclusion section outlines suggested model and discusses its possible applications.

\section{Governing Equations}

Considering an integrated description, within the upper highly turbulent ML of depth $h$, the density, $\rho_{m}$, temperature, $\theta_{m}$, salinity, $s_{m}$, and current velocity, $\boldsymbol{u}$, are constant. Below the ML, the interior is nonturbulent and continuously stratified, $\rho=\rho(z)$. The ML being highly turbulent, sharp density/temperature jumps must develop, with associated current velocity changes at its base, to express a marked separation from the nonturbulent interior layer.

In coordinate system traveling with the moving TC, stationary solutions are further considered. The TC moves opposite to $x_{1}$ direction with translation velocity $-V$ ( $V$ is positive value), and the TC eye coincides with $\left(x_{1}, x_{2}\right)$-coordinate origin. As such, partial time derivatives in all equations below are equivalent to 


$$
\partial / \partial t=V \partial / \partial x_{1}
$$

\subsection{Upper Mixed Layer}

Integrated over the ML depth, $h$, momentum and heat conservation equations read

$$
\begin{gathered}
h\left(\partial u_{\alpha} / \partial t+u_{\beta} \partial u_{\alpha} / \partial x_{\beta}+\varepsilon_{\alpha \beta} f u_{\beta}\right)=-g h \partial \zeta_{s} / \partial x_{\alpha}+\tau_{\alpha}^{s}-\tau_{\alpha}^{-h+0} \\
h\left(\partial \theta_{m} / \partial t+u_{\beta} \partial \theta_{m} / \partial x_{\beta}\right)=-q_{s}+q_{-h+0}
\end{gathered}
$$

where $\alpha, \beta=1,2$ are the indexes, $\zeta_{s}$ is the ocean surface displacement, $f$ is the Coriolis parameter, $\tau_{\alpha}^{s}$ and $q_{s}$ are the surface wind stress (scaled by water density) and kinematic heat fluxes, respectively, $\tau_{\alpha}^{-h+0}$ and $q_{-h+0}$ are the turbulent stress and heat flux at the ML base, $\varepsilon_{\alpha \beta}$ is the unit antisymmetric tensor $\left(\varepsilon_{\alpha \beta}=0\right.$ if $\alpha=\beta$ and $\varepsilon_{12}=-1, \varepsilon_{21}=1$ ), and $x_{3}$ axis is directed upward. The water density is defined by a known sea state law in the form $\rho=\rho_{0}(1+\alpha \theta+\beta s)$, where $\alpha$ and $\beta$ are thermal and haline expansion coefficients. As heat and salt balance equations are very similar, we solely consider the heat conservation equation, a corresponding equation for salinity being derived by replacing $\theta$ by $s$ and surface heat flux by overall resultant flux of the fresh water mass (precipitation minus evaporation). Turbulent stress and heat flux at the ML bottom, $x_{3}=-h+0$, are defined as

$$
\begin{aligned}
& \tau_{\alpha}^{-h+0}=u_{\alpha} w_{e} \\
& q_{-h+0}=-\Delta \theta w_{e}
\end{aligned}
$$

where $\Delta \theta=\theta_{m}-\theta_{-h-0}$ is the sharp temperature change at the ML base, $\theta_{-h-0}$ is the ocean temperature $\theta\left(x_{3}\right)$ below the ML at $x_{3}=-h-0$, and $w_{e}$ is the entrainment velocity defined as

$$
w_{e}=\partial h / \partial t+\partial M_{\beta}^{w} / \partial x_{\beta}
$$

where $\partial M_{\beta}^{w} / \partial x_{\beta}$ stands for the divergence of the total wind-driven transport, $M_{\beta}^{w}=h u_{\beta}$. Definition 4 applies as long as the ML is deepening/developing, that is, when $\partial h / \partial t+\partial M_{\beta}^{w} / \partial x_{\beta}>0$. In all other cases, $w_{e}=0$. Solely considering the linearized problem, use of equation (4) leads to the following momentum and heat balance equations:

$$
\begin{gathered}
\partial M_{\alpha}^{w} / \partial t+\varepsilon_{\alpha \beta} f M_{\beta}^{w}=-g h \partial \zeta_{s} / \partial x_{\alpha}+\tau_{\alpha}^{s} \\
\partial \theta_{m} / \partial t=-1 / 2 \bar{\Gamma} w_{e}-q_{s} / h
\end{gathered}
$$

where

$$
\bar{\Gamma}=\left(2 / h^{2}\right) \int_{0}^{h} z \Gamma \mathrm{d} z
$$

stands for the background (prestorm) temperature gradient averaged over the ML depth.

\subsection{Depth}

A review and developments of ML depth models can be found in Niiler (1975) and Zilitinkevich et al. (1979). Here we follow the assumption that ML deepens as to maintain a bulk Richardson number constant, that is, a critical $R i_{c}$ assumption. Considering the ML thickness, $h$, and sharp changes of buoyancy, $\Delta \rho$, and current velocity, $|\Delta \boldsymbol{u}|$, at the ML base, the critical, $R i_{c}$ is defined as

$$
R i_{c}=\frac{g\left(-\Delta \rho / \rho_{0}\right) h}{|\Delta \boldsymbol{u}|^{2}}
$$

Parametrization 8 had been widely used as to define the closure scheme for subgrid processes in numerical models simulating ocean response to TC passage, starting from the pioneering work by Price (1981) and further refined in Price et al. (1986). A sharp buoyancy jump at the ML base, $g \Delta \rho / \rho_{0}=g(\alpha \Delta \theta+\beta \Delta s)$, follows from the heat and salt balances within the ML. Ignoring effects of heat and salt surface fluxes, it comes 


$$
g\left(\Delta \rho / \rho_{0}\right)=-\frac{1}{2} h \overline{N^{2}}
$$

where $\overline{N^{2}}$ is the background (prestorm) Brunt-Väisälä frequency, $N^{2}$, averaged over the ML depth:

$$
\overline{N^{2}}=\left(2 / h^{2}\right) \int_{-h}^{0} z N^{2} \mathrm{~d} z
$$

Velocity sharp change, $|\Delta \boldsymbol{u}|$ in 8 , is associated to wind-driven velocities, $\boldsymbol{u}$, in the ML. Considering the magnitude of the volume wind-driven transport, $|M|=h|\boldsymbol{u}|$, the following relationship for the ML depth can be derived:

$$
h^{4}=2 R i_{c} \frac{|M|^{2}}{\overline{N^{2}}}
$$

Scaling $|M|$ as $|M| \propto u_{10}^{2} / f$, a classical relation for the ML depth in the stratified ocean is recovered, $h \propto u_{10} /$ $\sqrt{f N}$, (Pollard et al., 1972). For TC applications, the volume transport in equation (11) can be considered as a pure wind-driven transport, defined as solution of momentum balance equation (5) written in the rigid lid approximation:

$$
M\left(x_{1}, x_{2}\right)=f^{-1} \int_{x_{1}^{0}}^{x_{1}} \tau^{s}\left(x_{1}, x_{2}\right) \exp \left[-i k_{0}\left(x_{1}-x_{1}^{\prime}\right)\right] \mathrm{d}\left(k_{0} x_{1}^{\prime}\right)
$$

where $M=M_{1}+i M_{2}$ is the complex volume wind-driven transport, $k_{0}=f / V$ is the wave number of inertial oscillations, with boundary $\left(x_{1}^{0}, x_{2}\right)$ far ahead of the TC eye, where wind-driven currents induced by TC vanish, $M=0$.

\subsection{Interior Layer}

To treat the interior layer and its coupling with ML, we follow the model suggested by Orlanski and Polinsky (1983; see their section 2 for the details). The governing equation to describe the interior layer dynamics, $x_{3}<-h$, is the equation for vertical velocity, $w$. In the Fourier space, it reads

$$
\left(\Omega^{2}-f^{2}\right) \widehat{w}^{\prime \prime}-k^{2}\left(\Omega^{2}-N^{2}\right) \widehat{w}=0
$$

where hat over any quantity denotes Fourier transform, $\Omega=k_{1} V$ is the frequency, $k_{\alpha}$ is the wave number components, and, $k=\left(k_{1}^{2}+k_{2}^{2}\right)^{1 / 2}$, double prime indicates second derivative over the depth. This equation must be coupled with vertical velocity in the ML. Transforming the momentum balance (5), the coupling reads

$$
\left(\Omega^{2}-f^{2}\right)\left(\widehat{w}_{\mathrm{s}}-\widehat{w}_{\mathrm{h}}\right)-g h k^{2} \widehat{w}_{\mathrm{s}}=\widehat{F}
$$

where subscripts "s" and " $h$ " for $\widehat{w}$ denote vertical velocity at the surface and at the ML base, respectively, and $F$ is the wind stress forcing term

$$
F=f \cdot \operatorname{Rot}(\tau)-V \partial / \partial x_{1}[\operatorname{Div}(\tau)]
$$

combining vorticity, $\operatorname{Rot}(\tau)$, and divergence, $\operatorname{Div}(\tau)$, of surface wind stress field. Among these terms, only the vorticity term is capable to produce a steady state response in the ocean (Orlanski \& Polinsky, 1983). Hereinafter, the second term in (15) is therefore ignored, and Fsolely governed by the wind stress vorticity. Note that $w_{h}$ in equation (14) equals the divergence of the volume transport, $w_{h}=\partial M_{\beta}^{w} / \partial x_{\beta}$, and defines the entrainment velocity (4).

At $x_{3}=-h$ equations (13) and (14) are coupled through the kinematic, $\left.\widehat{w}\right|_{x_{3}=-h}=\widehat{w}_{h}$, and the dynamic, $\left.\left(\Omega^{2}-f^{2}\right) \widehat{w}^{\prime}\right|_{x_{3}=-h}=g k^{2}\left[\widehat{w}_{s}+(\Delta \rho / \rho) \widehat{w}_{h}\right]$, boundary conditions, where prime indicates derivative over the depth. At the bottom, $x_{3}=-H$, solutions must satisfy the boundary condition $\left.\widehat{w}\right|_{x_{3}=-H}=0$. 


\section{Simplification of Governing Equations}

Considering open ocean conditions, the ML depth is $\mathrm{O}(10-100 \mathrm{~m})$, small compared to both the ocean depth and the pycnocline depth, $D, \mathrm{O}(1,000 \mathrm{~m})$. Developing ML preserves mass, and the density jump at the ML base is also small, $\mathrm{O}(h / D)$, compared to the total density difference over the pycnocline. Consequently, the ML somehow appears as a small perturbation of the vertical ocean stratification, localized in a thin layer beneath the ocean surface. This significantly simplifies the problem in the following way.

As a first iteration, we can indeed decouple the interior layer dynamic from the ML. A baroclinic response can be readily estimated using the prestorm stratification condition, that is, without accounting for the small perturbations caused by the ML development and associated pycnocline erosion. At variance, the ML, and first of all its temperature, is strongly coupled with the interior layer through the entrainment velocity (4) affected by the upwelling velocity below the ML base. Thus, once the vertical velocity in the interior layer is determined, the system of ML heat balance equations, (6), (7), (4), and (11), is closed.

\subsection{Baroclinic Response}

\subsubsection{Three-Layer Approximation of Interior Layer}

To derive a practical analytical solution describing the baroclinic response, the vertical stratification in the interior layer is approximated by a three-layer model with constant $N$ in each of the layers: the seasonal, $x_{3}>-d$, and the main, $-D<x_{3}<-d$, pycnoclines with $N_{1}$ and $N_{2}$, respectively, and abyssal, $x_{3}<-D$, with $N_{3}=0$ (see Figure 9 in Kudryavtsev et al., companion paper). As tested, a three-layer approximation well fits general properties of the ocean vertical stratification, at least with a sufficient accuracy to adequately estimate TC-baroclinic responses. In addition, we assume that seasonal and main thermocline Brunt-Väisälä frequencies are much larger than the Coriolis parameter, $\left(N_{1}, N_{2}\right)>>f$. As TC-baroclinic responses are represented by near-inertial internal waves with $\Omega \approx f$, it also implies $\Omega<<\left(N_{1}, N_{2}\right)$.

\subsubsection{Solutions}

Solution of equation (13) for a three-layer approximation of the interior layer stratification, coupled with vertical velocity in the ML, equation (14), through boundary conditions, is detailed in equations (A.1) to (A.3). For typical ocean conditions, the ML depth is $\mathrm{O}(10-100 \mathrm{~m})$, and Brunt-Väisälä frequency in the seasonal pycnocline, $N_{1}$, is about $N_{1} \propto 10^{-2} 1 / \mathrm{s}$ or less. In this case, parameter $N_{1} h / C$, where $C$ is the phase velocity of long surface waves ( $C$ is about 2 to $3 \mathrm{~m} / \mathrm{s}$ ), is small, $N_{1} h / c=3 \times\left(10^{-2}\right.$ to $\left.10^{-1}\right)$. Solutions A.1 to A.3 with accuracy to small parameter $1 / 2\left(N_{1} h / c\right)^{2}<<1$ can be simplified to relations (A.6)-(A.7), not explicitly dependent on the ML parameters. This fact suggests that while entirely driven by divergence of wind currents in the ML, the vertical structure of baroclinic motions is not strongly affected by small perturbations of the ocean stratification associated with the ML. Accordingly, the baroclinic response can effectively be calculated using prestorm stratification conditions.

The vertical velocity of baroclinic motions of the $n$ mode in the seasonal and the main pycnocline can be written as (see section $\mathrm{b}$ in Appendix for details)

$$
w_{n}(\boldsymbol{x}, z)= \begin{cases}a_{1 n} W_{n}(\boldsymbol{x}) \sin \left[N_{1} x_{3} / C_{n}\right], & \text { at }-d<x_{3}<0 \\ a_{2 n} W_{n}(\boldsymbol{x}) \sin \left[N_{2}\left(D+x_{3}\right) / C_{n}+\varphi_{n}\right], & \text { at }-D<x_{3}<-d\end{cases}
$$

and in the abyssal, $-H<x_{3}<-D$, it linearly varies with depth, as

$$
w_{n}(\boldsymbol{x}, z)=w_{n}(\boldsymbol{x}, D)\left(H+x_{3}\right) /(H-D)
$$

In these relations, subscript "n" for any quantity indicates the mode number, $C_{n}$ is the phase velocity of long internal waves of the $n$ mode defined from the dispersion relationship:

$$
\sin \left[\frac{N_{1} d}{C_{n}}+\frac{N_{2}(D-d)}{C_{n}}+\varphi_{n}\right]-\frac{N_{1}-N_{2}}{N_{1}+N_{2}} \sin \left[\frac{N_{1} d}{C_{n}}-\frac{N_{2}(D-d)}{C_{n}}-\varphi_{n}\right]=0
$$

with $\sin \varphi_{n}=\left[N_{2}(H-D) / C_{n}\right] / \sqrt{1+\left[N_{2}(H-D) / C_{n}\right]^{2}}$ (for the deep ocean $\varphi=\pi / 2$ ), $a_{1 n}$ and $a_{2 n}$ are dimensionless vertical velocity amplitudes defined by A.13, and $W_{n}(\boldsymbol{x})$ is a function defining $2 \mathrm{D}$ field of vertical velocity for $n$ mode: 


$$
W_{n}\left(x_{1}, x_{2}\right)=\frac{1}{4 \pi^{2} C_{n}^{2}} \iint \frac{\widehat{F}\left(k_{1}, k_{2}\right)}{\left(V^{2} / C_{n}^{2}-1\right) k_{1}^{2}-k_{2}^{2}-\kappa^{2}} e^{i k_{\beta} x_{\beta}} \mathrm{d} k_{1} \mathrm{~d} k_{2}
$$

where $\kappa=f / C_{n}$ is the inverse baroclinic radius of deformation. If the TC-translation velocity is large enough to satisfy $V>C_{n}$, relation (19) exhibits a singularity around a resonant curve in the wave number space: $k_{1}^{2}$ $\left(V^{2} / C_{n}^{2}-1\right)-k_{2}^{2}-\kappa^{2}=0$.

An analytical solution for this case is given by Geisler (1970, his equation (30)). For our purpose, a slightly different form of the solution is suggested to be numerically estimated using fast Fourier transform. This solution reads (see section $\mathrm{b}$ in Appendix for details):

$$
W_{n}\left(x_{1}, x_{2}\right)=\frac{1}{2 \pi\left(V^{2}-C_{n}^{2}\right)} \int_{-\infty}^{x} \mathrm{~d} x_{1}^{\prime} \int_{-\infty}^{\infty} \widehat{F}\left(x_{1}^{\prime}, k_{2}\right) \frac{\sin \left(k_{01}\left(x_{1}-x_{1}^{\prime}\right)\right)}{k_{01}} e^{i k_{2} x_{2}} \mathrm{~d} k_{2}
$$

where $\widehat{F}\left(x_{1}^{\prime}, k_{2}\right)$ is the $k_{2}$ Fourier transform of wind stress source for a given $x_{1}^{\prime}$, and $k_{01}$ is the resonant wave number defined by A.17. For slow TC, $V<C_{n}$, relationship (19) does not possess any singularity, and the vertical velocity is found directly as Fourier transform.

Solution 20 for fast TC, $V>C_{n}$, and solution 19 for slow TC, $V<C_{n}$, together with 16 to 18 provide a full description of the TC-baroclinic vertical motions. Given vertical velocities, displacements of the water masses at a given depth, $\zeta\left(\boldsymbol{x}, x_{3}\right)$, are then straightforwardly determined as the integral of vertical velocity over the horizontal space:

$$
\zeta_{n}\left(\boldsymbol{x}, x_{3}\right)=V^{-1} \int_{-\infty}^{x_{1}} w_{n}\left(\boldsymbol{x}, x_{3}\right) \mathrm{d} x_{1}
$$

Correspondingly, total displacement is to be found as superposition of the different modes.

\subsection{Surface Height Anomalies Induced by TC}

Ginis and Sutyrin (1995) argued that barotropic and baroclinic modes do not interact and can thus independently be considered. The TC-induced total sea surface height (SSH) anomalies, $\zeta_{s}$, thus writes as a sum of baroclinic, $\zeta_{s}^{b c}$, and barotropic, $\zeta_{s}^{b t}$, components: $\zeta_{s}=\zeta_{s}^{b c}+\zeta_{s}^{b t}$. To second order of the small parameter $N_{1} h / C_{n}$, the surface vertical velocity associated to the $n$ mode of the baroclinic motions follows from boundary conditions and reads as

$$
w_{s n}^{b c}(\boldsymbol{x})=\left.\left(C_{n}^{2} / g\right) w_{n}^{\prime}\right|_{x_{3}=0}=a_{1 n}\left(N_{1} C_{n} / g\right) W_{n}(\boldsymbol{x})
$$

The corresponding SSH anomalies, $\zeta_{S}$, follow from 21 with 22.

The barotropic component of the surface vertical velocity is taken into account in the governing equations (13) and (14). The barotropic mode is not sensitive to the ocean stratification, attenuates linearly with the depth, and the maximum of vertical velocity occurs at the surface. Evaluating $w_{h}^{\prime}$, as $\widehat{w}_{h}^{\prime}=\widehat{w}_{h} /(H-h)$, expressing then $\widehat{w}_{h}$ via $\widehat{w}_{s}$, and substituting it to (14), we arrive at equation

$$
\widehat{w}_{s}^{b t}\left[\left(V^{2} / C_{b}^{2}-1\right) k_{1}^{2}-k_{2}^{2}-f^{2} / C_{b}^{2}\right]=\widehat{F} / C_{b}^{2}
$$

which is similar to A.14, except that $C_{b}$ corresponds to the phase velocity of long surface waves: $C_{b}^{2}=g H$. Since $C_{b}>>V$, barotropic vertical velocity can be directly found from 23 as inverse Fourier transform:

$$
w_{s}^{b t}\left(x_{1}, x_{2}\right)=-1 /\left(4 \pi^{2} g H\right) \iint \widehat{F} /\left(k^{2}+\kappa_{b t}^{2}\right) e^{i k_{\beta} x_{\beta}} \mathrm{d} k_{1} \mathrm{~d} k_{2}
$$

where we ignored $V$, very small compared to $C_{b}$, and $\kappa_{b t}=f / C_{b}$ is the inverse barotropic radius of deformation. Correspondingly, the SSH anomalies caused by the barotropic mode can be calculated using 21 with 24 .

\subsection{Heat Balance in the Upper Mixed Layer}

As considered, the three-layer model has been introduced to derive tractable analytical solutions for the baroclinic motions. Deviations of the real stratification from a three-layer approximation should not 


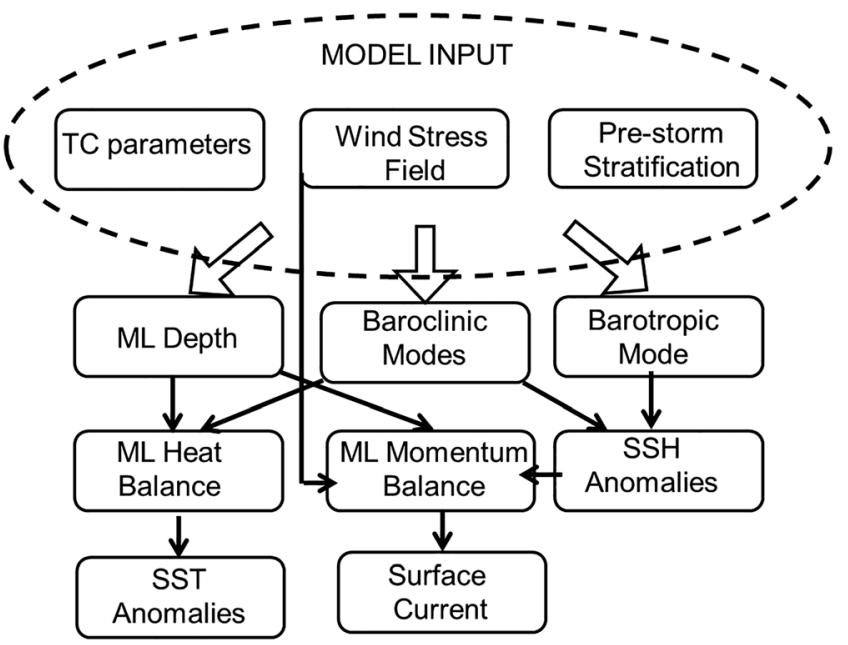

Figure 1. Flow diagram of the model. quantitatively impact the main characteristics of the baroclinic modes. At variance, the ML heat balance, equation (6), and its depth, equation (11), drastically depend on the stratification in the upper ocean. Thus, to simulate the ML and the SST wake, realistic ocean stratification must be considered. As discussed in section 6.2, the ML evolves so that

$$
R i=(1 / 2) h^{4} \overline{N^{2}} / M_{m}^{2}
$$

keeps a critical value, $R i=R i_{c r}$, see equation (11). This may only be valid for deepening and/or stationary conditions. The ML deepening is an irreversible process. Therefore, the ML depth must not decrease when the total wind-driven transport/stratification is locally reduced/enhanced. For such conditions, the ML depth either keeps a constant value or undulates following vertical movements: $\partial h / \partial t=-w_{h}$. Following such reasoning, the entrainment velocity at the base of the ML is defined as

$$
w_{e}= \begin{cases}V \partial h / \partial x_{1}+w_{h}, \text { if } R i=R i_{\mathrm{cr}} \\ 0, & \text { if } R i>R i_{\mathrm{cr}}\end{cases}
$$

During the forced stage cooling/warming of the ML due to the surface heat fluxes plays a secondary role (Price et al., 1994); therefore, term $q_{s} / h$ in 6 can be ignored. Surface heating of the TC cold wake acts on time scale of order few days. Equation (6) thus simplifies to

$$
\partial \theta_{m}\left(x_{1}, x_{2}\right) / \partial x_{1}=-1 / 2 \bar{\Gamma} w_{e} / V
$$

or in terms of the SST anomalies, $\delta \theta_{S}$ :

$$
\delta \theta_{s}\left(x_{1}, x_{2}\right)=-1 / 2 V^{-1} \int_{x_{10}}^{x_{1}} \bar{\Gamma} w_{e} \mathrm{~d} x_{1}^{\prime}
$$

This relation together with $26,7,11$ and vertical velocity 16 taken at the base of the ML completes the ML description.

Flow diagram, Figure 1, demonstrates links between different components of the model.

\section{Model Results}

To specify the wind forcing, the radial wind speed profile can follow the form suggested by Holland (1980), as

$$
u(r)=\left[\left(u_{m}^{2}+u_{m} r f\right)\left(\frac{R_{m}}{r}\right)^{B} \exp \left(-\left(\frac{R_{m}}{r}\right)^{B}+1\right)+\left(\frac{r f}{2}\right)^{2}\right]^{1 / 2}-\frac{r f}{2}
$$

where $u_{m}$ is the maximum wind speed at $10-\mathrm{m}$ level, $R_{m}$ is the radius of maximum wind speed, and $B$ defines the shape of the wind field with increasing radial distance $r$.

Below we present some results, considering the following parameters, typical of ocean environmental conditions: $N_{1}=1.36 \times 10^{-2} 1 / \mathrm{s}, N_{2}=3.9 \times 10^{-3} 1 / \mathrm{s}, d=200 \mathrm{~m}, D=1,000 \mathrm{~m}$, and $H=5,000 \mathrm{~m}$. Wind speed is specified by 29 , and it is assumed that wind velocity spirals toward the TC eye, with a constant inflow angle of $25^{\circ}$ (Shea \& Gray, 1973), and $R_{m}=50 \mathrm{~km}, u_{m}=50 \mathrm{~m} / \mathrm{s}$, and $B=1.5$. The wind stress acting to the ocean surface is

$$
\tau_{\alpha}^{s}=\left(\rho_{a} / \rho_{w}\right) C_{d} u u_{\alpha}
$$

where $C_{d}$ is drag coefficient, $u$ is wind speed at reference level (e.g., $10 \mathrm{~m}$ ), and $u_{\alpha}$ is wind velocity component. Parameterization of the drag coefficient at high wind speeds is still a matter of debate. As generally recognized, the relation for $C_{d}$, valid for moderate winds, must not be extrapolated to hurricane-force wind conditions. Scanty amount of observations demonstrates that $C_{d}$ levels off and/or falls at wind speeds above 

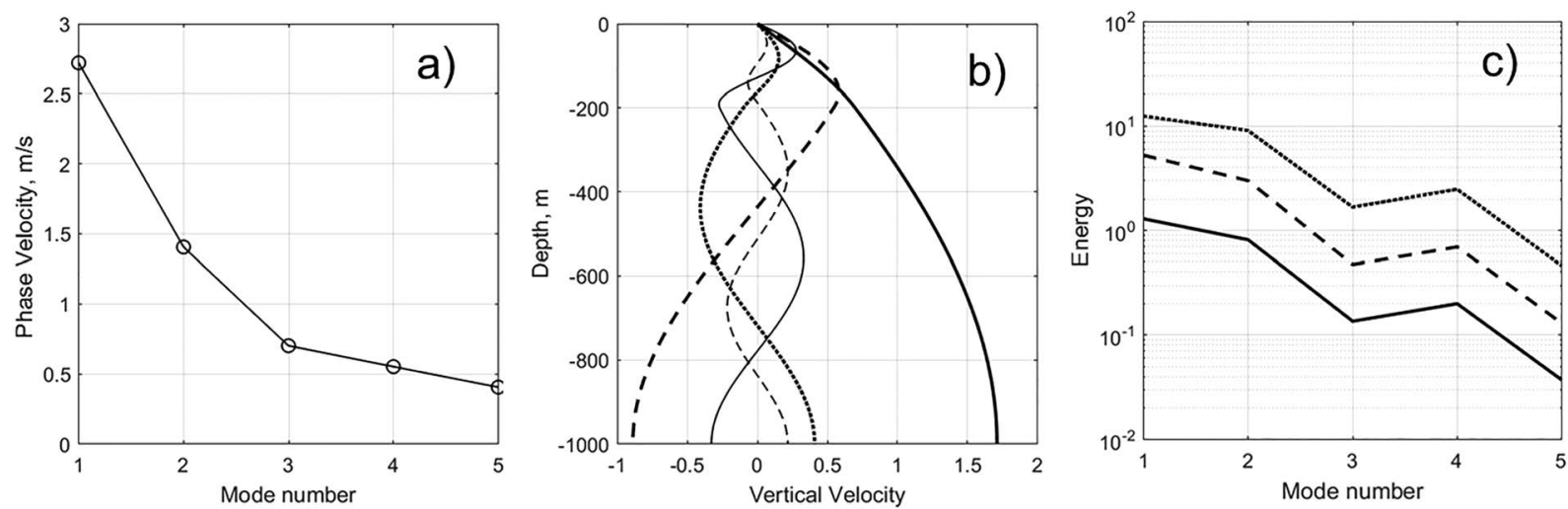

Figure 2. (a) Dispersion relation (18) for the five first modes; (b) profiles of vertical velocity for the five first modes: thick solid, dashed, and dotted lines, and thin solid and dashed lines, respectively; (c) redistribution of energy (conventional units) between baroclinic modes generated by TC traveling with translation velocities: (solid) $V=10 \mathrm{~m} / \mathrm{s}$, (dashed) $V=5 \mathrm{~m} / \mathrm{s}$, and (dotted) $V=2.5 \mathrm{~m} / \mathrm{s}$. TC $=$ tropical cyclone.

$30 \mathrm{~m} / \mathrm{s}$ (e.g., Powell et al., 2003; also see Figure 14 in the companion paper). For our demonstration purpose, the surface drag coefficient and wind stress parametrizations follow those suggested in Kudryavtsev et al. (companion paper).

\subsection{Baroclinic and Barotropic Responses}

Phase velocities of long IW, using dispersion relation (18) and vertical structure for the first five modes, are shown in Figure 2. The low limit of the depth axis in Figure $2 \mathrm{~b}$ corresponds to the depth of the main pycnocline base, $x_{3}=-D$; hence, vertical velocity of each of the modes at $x_{3}<-D$ attenuates linearly to meet boundary condition $w=0$ at $x_{3}=-H$. Figure 2 c illustrates the redistribution of the (conventional) energy

$$
E(n)=\overline{\int_{-D}^{0} N^{2} \zeta_{n}^{2} \mathrm{~d} x_{3}}
$$

of the baroclinic motions, between the different modes triggered by a moving TC; $\zeta_{n}$ in 31 is the pycnocline displacement for each mode defined by 21. From Figure 2c, most part of the energy of baroclinic motions is contained in the two first baroclinic modes. Similar results (not shown) are generally found for other TC parameters and ocean stratification conditions. Therefore, we only consider the baroclinic response as the composition of these two lowest baroclinic modes.

Figure 3 illustrates the $2 \mathrm{D}$ fields of vertical velocities, equation (19), generated by TC, either fast $(V=5 \mathrm{~m} / \mathrm{s})$ relative to both IW modes, $V / C_{1}=1.84$ and $V / C_{2}=3.56$, or $\operatorname{slow}(V=2 \mathrm{~m} / \mathrm{s})$ relative to the first mode, $V / C_{1}=$ 0.74 , but fast relative to the second one, $V / C_{2}=1.42$. If the $\mathrm{TC}$ is fast, it generates wake of near-inertial IWs (Geisler, 1970). The wave number of these waves is about $k_{0}=\left(f / C_{n}\right) / \sqrt{V^{2} / C_{n}^{2}-1}$, due to their dispersive nature, and the amplitude of the vertical velocity attenuates with the distance from the TC eye (along the central line, $\left.x_{2}=0\right)$ as $\propto\left(k_{0} x_{1}\right)^{-1 / 2}$. In the wake, IWs populate a wedge with half-angle, $\beta$, equal to $\tan \beta=\left(V^{2} / C_{n}^{2}-1\right)^{-1 / 2}$. Thus, the faster is the TC, the longer is the IW length and narrower will be the wedge filled by these IWs. If a TC is slow relative to the first mode, $V / C_{1}<1$, it does not generate IW wake behind, but it causes a localized upwelling, spatially confined to the area of the surface stress vorticity action, Figure 3c.

Baroclinic and barotropic components of the SSH anomalies are shown in Figure 4. For a fast TC, baroclinic SSH anomalies are formed by the IW wake, with a dominant contribution of the first mode. Barotropic SSH anomaly has a shape of the surface trough, with a depth much smaller than the baroclinic SSH anomaly contribution. This derives from considering deep ocean conditions. For shallow ocean conditions, relations between magnitudes of the barotropic and the baroclinic SSH anomalies could very well be opposite. For slow TC, with $C_{2}<V<C_{1}$, baroclinic SSH anomaly is a composition of the surface trough caused by a nonresonant upwelling contribution from the first mode overlapped with high-frequency IW oscillations. Similar to fast TC, barotropic SSH anomalies for slow TC are significantly smaller than the baroclinic ones. The total 

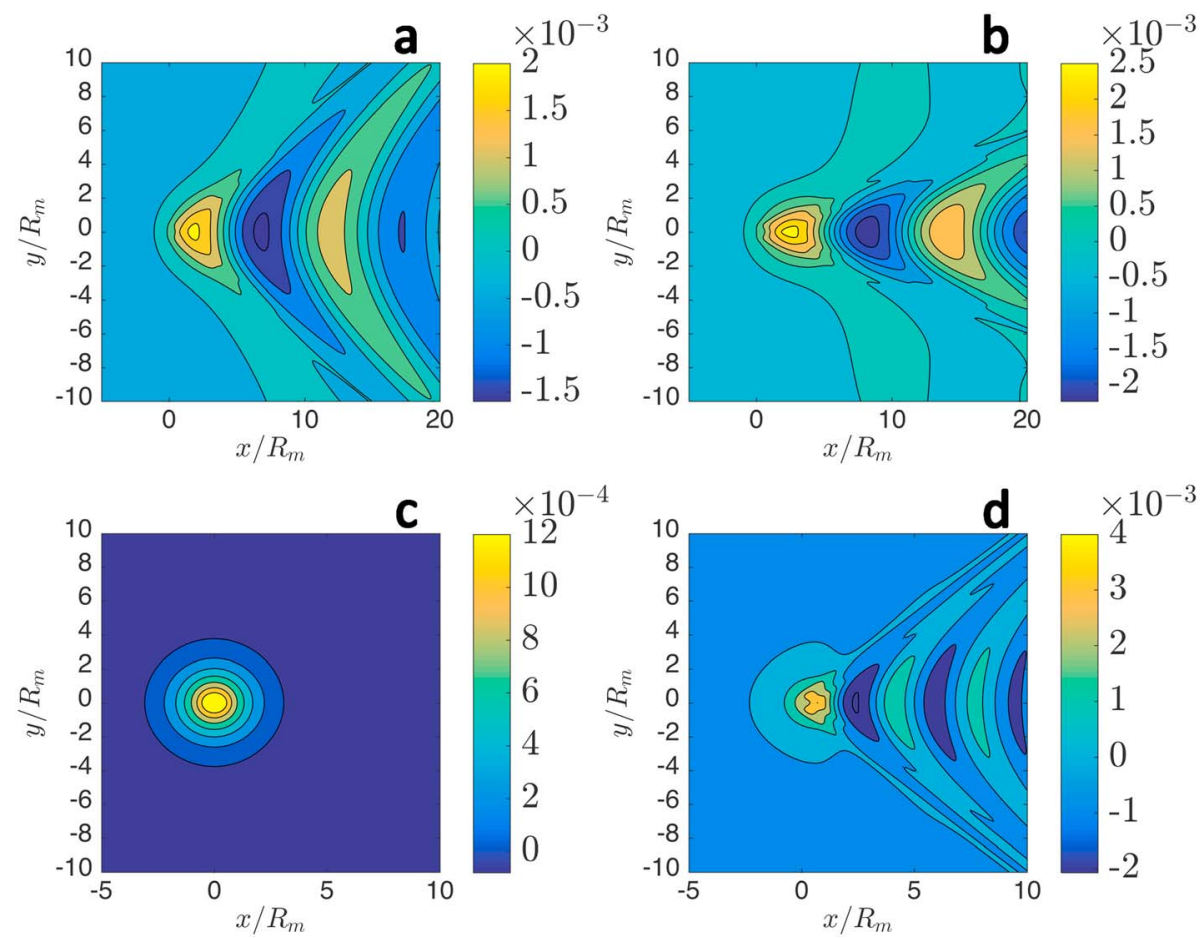

Figure 3. Two-dimensional field of vertical velocity described by 19 for $(a, c)$ the first and $(b, d)$ the second baroclinic modes generated by TC traveling with translation velocity (a, b) $V=5 \mathrm{~m} / \mathrm{s}$ and (c, d) $V=2 \mathrm{~m} / \mathrm{s}$. Color bar in each of the plots indicates velocity in meter per second. $\mathrm{TC}=$ tropical cyclone.

SSH anomalies (sum of baroclinic and barotropic components) are shown in Figures 5a and 5b. Comparing magnitudes of SSH anomalies induced by fast and slow TC, one may find that the SSH anomalies are more marked for a slow TC.

Once the SSH anomalies are found, the sea surface current field can be determined through solution of the integral momentum balance equation for the ML, equation (5), with prescribed momentum source-sum of the surface wind stress and the pressure gradient. Solution of this equation is straightforward and has the form similar to equation (12), where, however, the wind stress under the integral must be replaced by momentum source (equal to the right side of equation (5)). Then, with known ML depth, the surface currents, $u_{\beta}$, read $u_{\beta}=M_{\beta}^{w} / h$. Fields of the surface current for fast and slow TCs are shown in Figures $5 \mathrm{c}$ and $5 \mathrm{~d}$ correspondingly. These fields are composed of superposition of the wind-driven currents and currents induced by the baroclinic and the barotropic motions.

The model SSH anomalies, scaled by $u_{m}^{2} / g$, as a function of dimensionless parameter $R_{m} N_{1} / V$ characterizing the properties of the TC and the upper ocean stratification, are shown in Figure 6. An empirical relation $g h_{s} / u_{m}^{2}=6.9 \times 10^{-6}\left(R_{m} N_{1} / V\right)$ suggested from satellite SSH measurements (see Figure 14 in Kudryavtsev et al., companion paper) is also shown. As found, the model is consistent with reported observations. Yet the model seems to indicate that dimensionless SSH anomalies are not fully self-similar on parameter $R_{m} N_{1} / V$.

\subsection{SST Wake}

Figure 7 evidences the impact of the baroclinic wake on the 3D field of the ocean temperature, for a fast TC with $V=5 \mathrm{~m} / \mathrm{s}$, Rossby number $V / f R_{m}=2$ and $V>C_{1}=2.7 \mathrm{~m} / \mathrm{s}$, and a slow TC with $V=2 \mathrm{~m} / \mathrm{s}$, Rossby number less than $1, V / f r_{m}=0.8$, and $C_{1}>V>C_{2}=1.4 \mathrm{~m} / \mathrm{s}$.

In both cases, the SST wake has a remarkable bias to the right side of the TC track, stronger for the faster TC condition. This can be attributed to resonant couplings between the rotation of surface winds and clockwise inertial currents, accelerated (respectively decelerated) on the right side (respectively left side). ML stirring and entrainment from below the thermocline are thus amplified (Huang \& Oey, 2015; Price, 1981; Skyllingstad et al., 2000). 

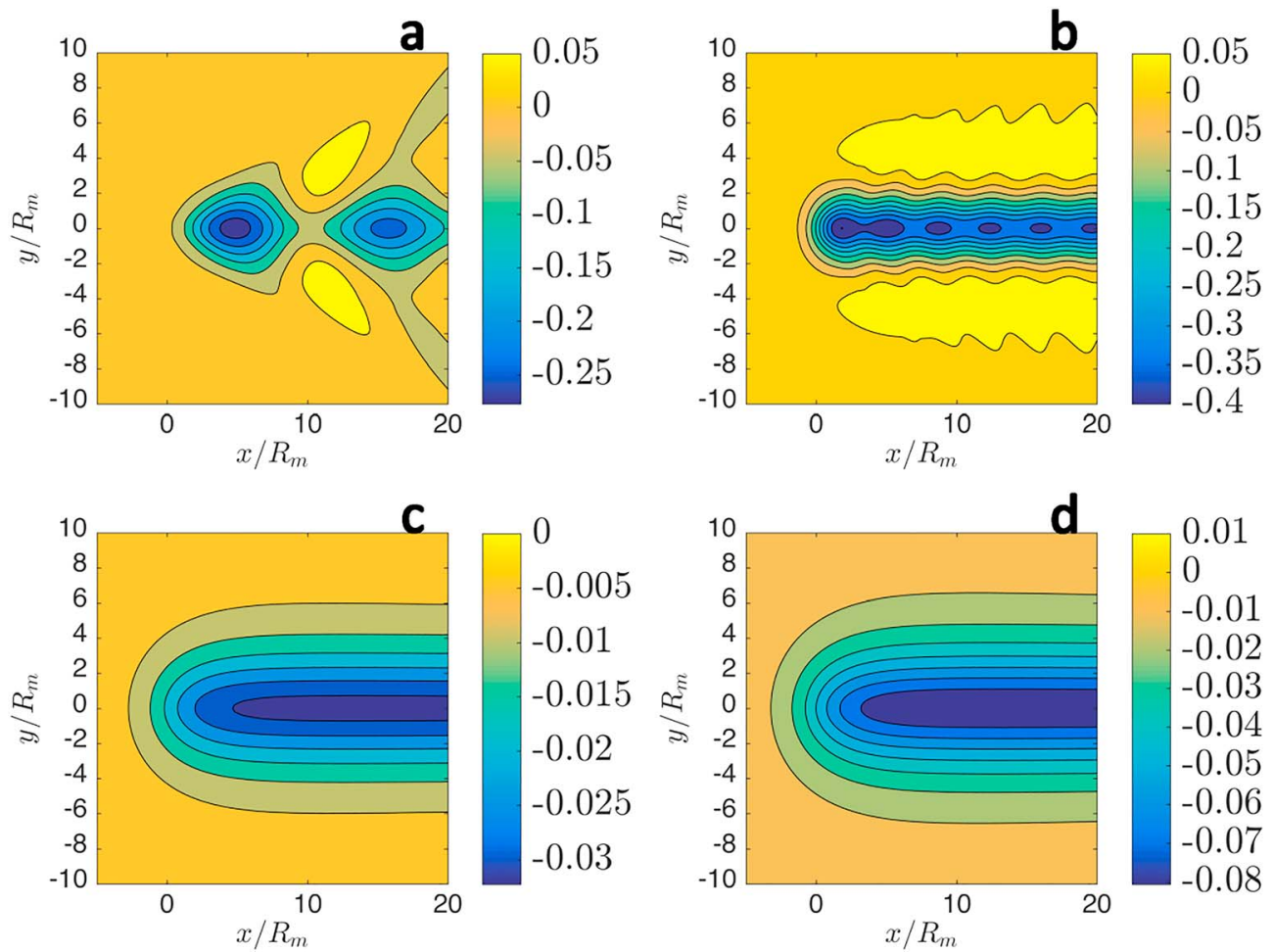

Figure 4. (a, b) Baroclinic and (c, d) barotropic components of the sea surface height anomalies generated by TC traveling with translation velocity (a, c) $V=5 \mathrm{~m} / \mathrm{s}$ and (b, d) $V=2 \mathrm{~m} / \mathrm{s}$. Color bar in each of the plots indicates SSH anomalies in meter. $\mathrm{SSH}=$ sea surface height.

Vertical transects, along the TC track, reveal two stages of the SST wake development: a forced stage $\left(x_{1} /\right.$ $R_{m}<5$ for fast TC and $x_{1} / R_{m}<2$ for slow TC in Figures $7 \mathrm{c}$ and $7 \mathrm{~d}$ correspondingly), characterizing deepening of the ML and its cooling due to heat fluxes caused by both the thermocline erosion (first term in equation (26), upper line) and upwelling (second term in equation (26), upper line), followed by a relaxation stage at larger $x_{1} / R_{m}$ values. At this stage, turbulent mixing at the ML base ceases, $R i>R i_{\mathrm{cr}}$. ML evolves keeping constant temperature, as surface heat fluxes are not taken into account, and its lower boundary undulates following quasi-inertial IW oscillations, equation (26)—lower line.

Vertical transects, across the TC track, Figures 7e and 7f, are related to the forced stage of the ocean response. The ML depth is controlled by the magnitude of the wind-driven transport, with a clear bias to the right of the track. The upwelling effect lifts up isotherms to impale the ML boundary, enhancing cooling of the upper layer.

Model SST anomalies, with and without accounting for the upwelling impact, scaled by $\left\langle\delta \theta_{m}\right\rangle=2 \Gamma u_{m} /$ $\left(f N_{1}\right)^{1 / 2}$ are shown in Figure 8, as a function of the TC-Rossby number. As expected, taking into account upwelling remarkably impacts the SST anomalies, especially for low translation velocities. Effect of the upwelling on SST anomalies is also illustrated in Figure 18a below. The scaled SST anomalies are not selfsimilar on the TC Rossby number $R o \equiv V /\left(f R_{m}\right)$, that is, model curves do not converge for different winds. Similar conclusions could be drawn from varying the radius of maximal wind speed, the shape parameter, and parameters related to the ocean stratification. Strictly, the model results do not fully support a selfsimilarity of the dimensionless anomalies on TC Rossby number, as initially suggested from the analysis of observations (Kudryavtsev et al., companion paper).

To first order, the empirical parametrization for the scaled SST anomalies (thick gray line in the right plot of Figure 8) does not perfectly compare with initial model simulations. Indeed, initial setups consider very idealized stratifications of the upper layer, with a constant temperature gradient up to the ocean surface. More likely, stratification of the upper top ocean shall be different, with rather uniform vertical temperature profiles, see, for example, Figure 9 from (Kudryavtsev et al., companion paper). As derived, equation (28) with 7 , 


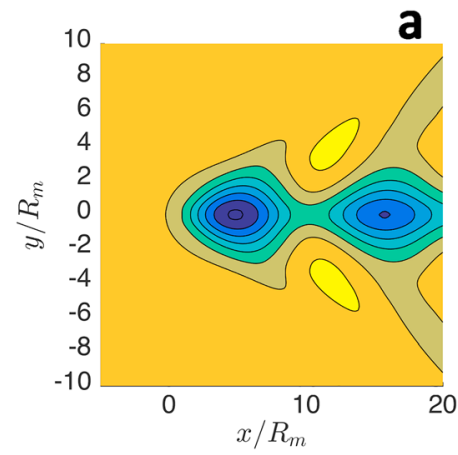

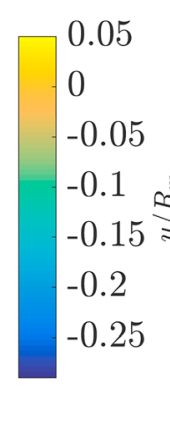

b
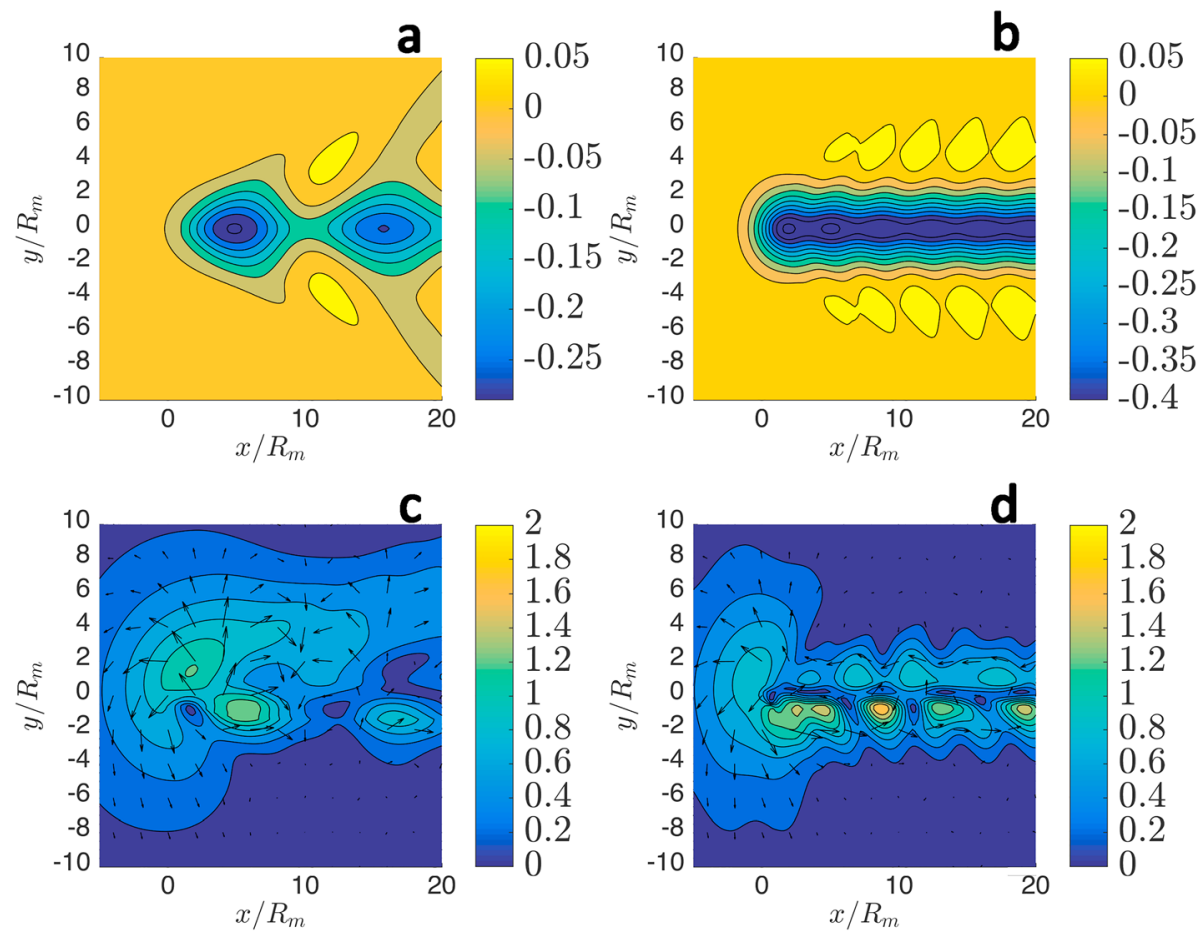

0.05

$-0.1$

$-0.15$

$-0.2$

$-0.25$

$-0.3$

$-0.35$

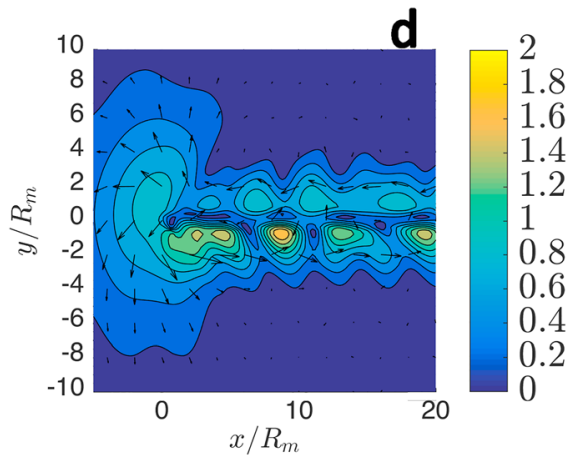

Figure 5. (a, b) The total (sum of baroclinic and barotropic components) sea surface height anomalies and (c, d) surface currents generated by TC traveling with translation velocity (a, c) $V=5 \mathrm{~m} / \mathrm{s}$ and (b, d) $V=2 \mathrm{~m} / \mathrm{s}$. Color bar in each of the plots indicates SSH anomalies in meter and surface current speed in meter per second correspondingly. Arrows indicate direction of the current velocity.

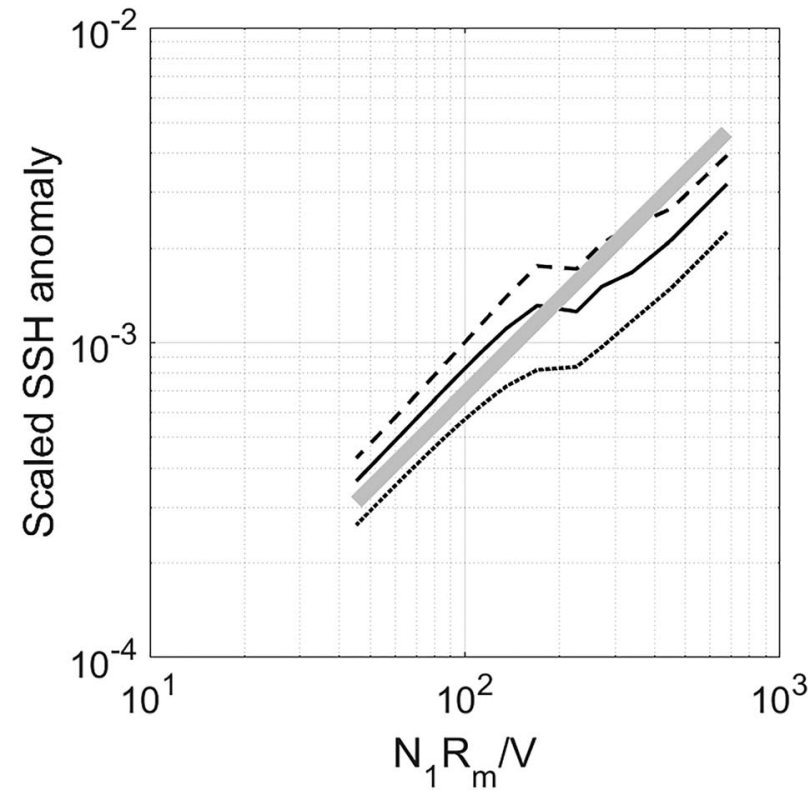

Figure 6. Dependence of dimensionless model SHH anomalies $g h_{s} / u_{m}^{2}$ on parameter $R_{m} N_{1} / V$ for different maximal wind speed: (dashed) 30, (solid) 50 , and (dotted) $70 \mathrm{~m} / \mathrm{s}$. Thick gray line is empirical relation $g h_{s} / u_{m}^{2}=6.9 \times$ $10^{-6}\left(R_{m} N_{1} / V\right)$ suggested by Kudryavtsev et al. (companion paper). TC parameters: $R_{m}=50 \mathrm{~km}, 1 \mathrm{~m} / \mathrm{s}<V<15 \mathrm{~m} / \mathrm{s}$. SSH = sea surface height; $\mathrm{TC}=$ tropical cyclone.
SST anomalies are very sensitive to this vertical distribution of the temperature. To illustrate this property, the prestorm stratification can be assumed as a composition of a constant temperature subsurface layer, with thickness, $h_{0}$, much smaller than the depth of seasonal thermocline, $h_{0}<<d$, superposed over a lower layer with constant temperature gradient. The averaged temperature gradient 7 , defining an SST anomaly via 28 , is then reduced by factor $\left(1-h_{0}^{2} / h^{2}\right)$. As the sensitivity for the ML depth, $h$, to the temperature gradient is much weaker, power one fourth of the gradient, see equation (11), the SST anomalies in presence of an upper uniform layer can be simply estimated by multiplying the reference SST anomalies (for constant temperature gradient) by the factor $\left(1-h_{0}^{2} / h^{2}\right)$. As obtained, Figure $8 \mathrm{c}$, taking into account this uniform upper layer effect, largely widens the range of model estimates to encompass the empirical relation.

\section{Simulations of Observations}

In this section, simulations are performed to compare with satellite observations, SST, and SSH anomalies, reported in Kudryavtsev et al. (companion paper).

\subsection{Input Parameters}

The best-track (BT) data describing key TC characteristics, that is, maximum wind speed and its radius, radii of given wind speed and translation velocity (derived from TC 6-hr position) for three TC were reported (Reul et al., 2017). To perform the model simulations, these input characteristics 

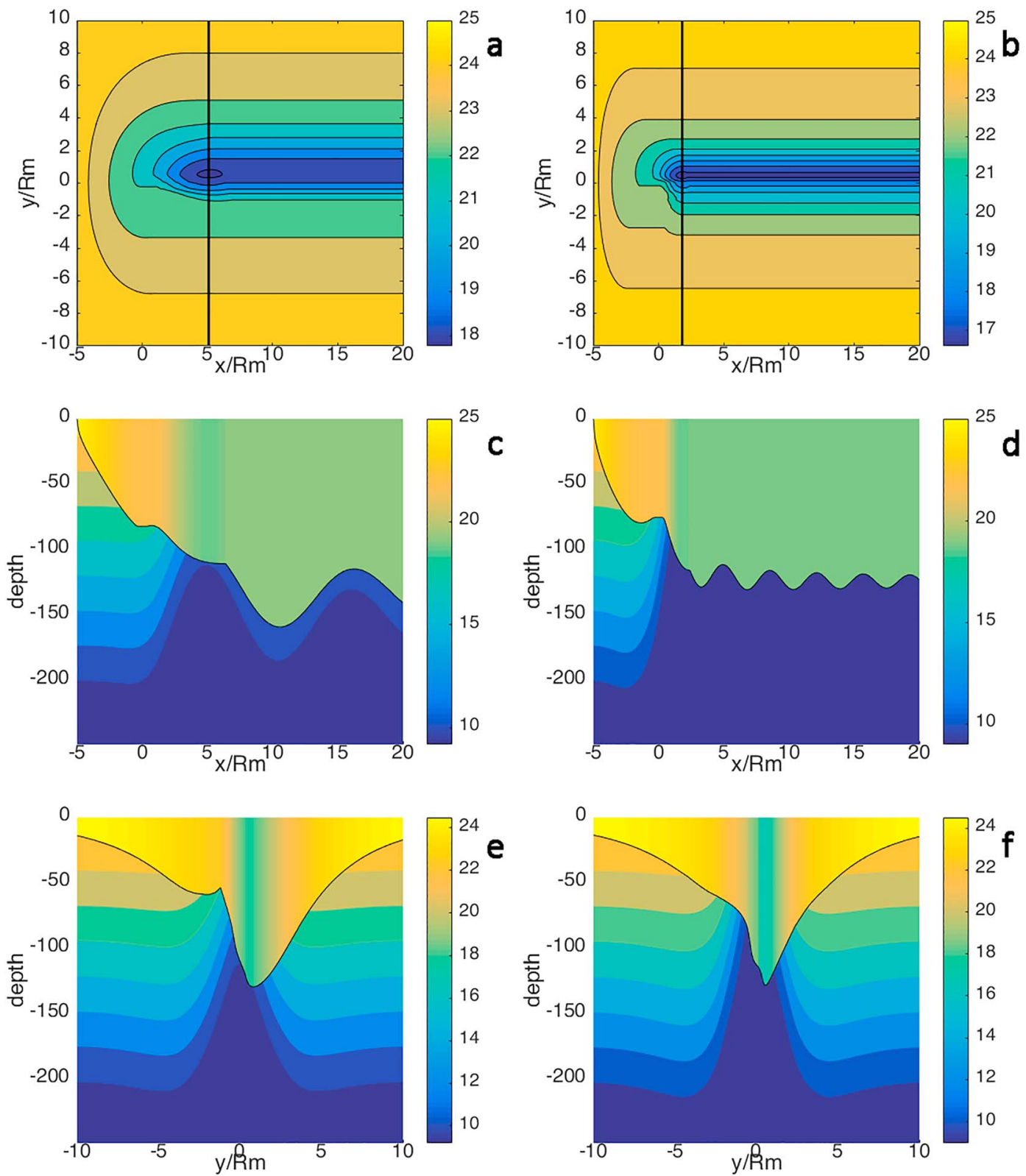

Figure 7. SST wake generated by (a, c, e) fast and (b, d, f) slow TC with translation velocity $V=5$ and $V=2 \mathrm{~m} / \mathrm{s}$ correspondingly. (a, b) SST fields (view from the top), (c, d) $x_{1}-x_{3}$ transects at $x_{2}=0$, and (e, f) $x_{2}-x_{3}$ transects at $x_{1}$ indicated in the corresponding upper plots. SST $=$ sea surface temperature.

are averaged over a 1-day sliding window, Figure 9 . The BT radial wind speed distributions were then fitted to the model wind speed 29. The maximum wind speed, $u_{m}$, is fixed, and the two other model parameters are adjusted, $R_{m}$ and $B$ (see Figure 9), by minimizing the difference between the wind model and the radial velocity distribution averaged over TC sectors. Two examples of this procedure, demonstrating good agreement for the corresponding radial distributions of wind stress and its vorticity, are shown in Figure 10.

To specify the ocean stratification along the TC tracks, the WORLD OCEAN ATLAS 2013 version 2 (https:// www.nodc.noaa.gov/OC5/woa13/) is used. As a comparative ground-truth data set, ISAS-15 gridded monthly fields of temperature and salinity based on ARGO profilers is also employed (Kolodziejczyk et al., 2017). For the evolution of the ML depth and SST anomalies, simulations are performed using real 

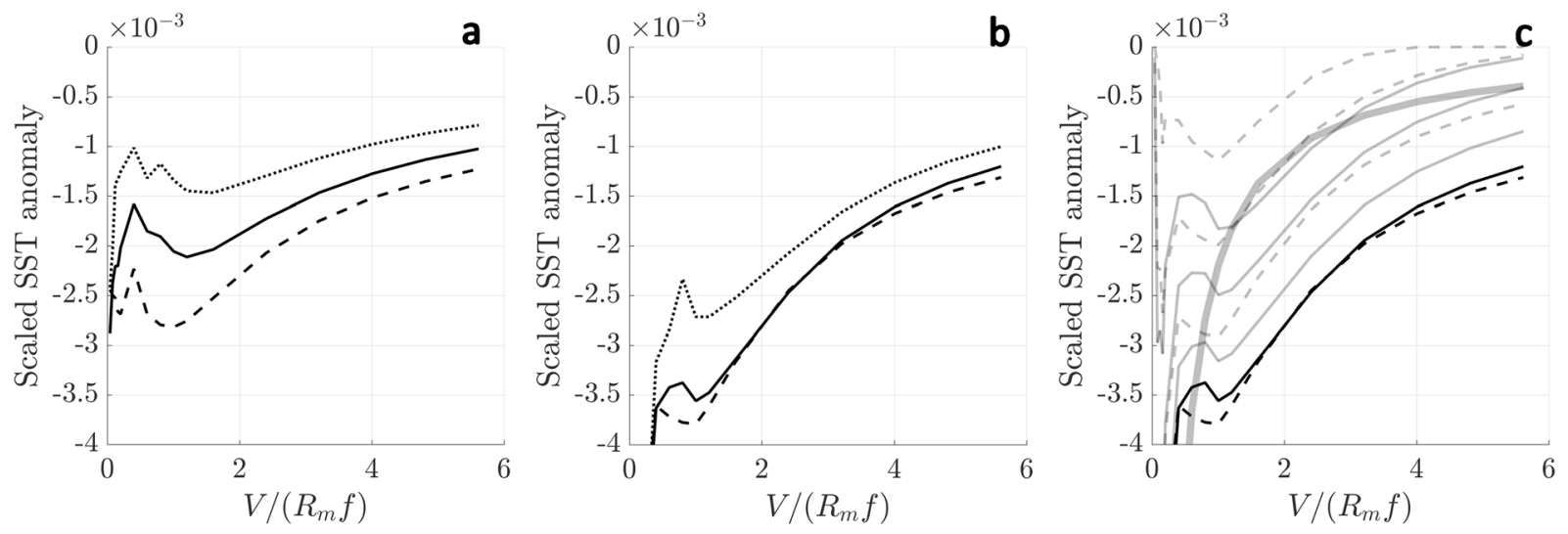

Figure 8. Normalized SST anomalies, $\widehat{\delta \theta}_{m}=2\left(\delta \theta_{m} / \Gamma\right)\left(f N_{1}\right)^{1 / 2} / u_{m}$, as a function of TC Rossby number, $V / f R_{m}$, at different maximum wind speeds: (dashed) 30 , (solid) 50, and (dotted) $70 \mathrm{~m} / \mathrm{s}$. Plots (a) and (b) show simulations when impact of upwelling on SST wake is either taken or not taken into account. Plot (c) illustrates the impact of a prestorm upper layer of uniform temperature with thickness 0 (thick lines), 20, 40, and $60 \mathrm{~m}$ (thin lines of the same style from down to top, respectively) on the SST anomalies at (dashed lines) 30 and (solid lines) $50 \mathrm{~m} / \mathrm{s}$. In these calculations $R_{m}=50 \mathrm{~km}, f=5 \times 10^{-5} 1 / \mathrm{s}$. Thick gray line in the right plot shows parametrization of the SST anomalies suggested from observations (Kudryavtsev et al., companion paper). SST $=$ sea surface temperature; TC $=$ tropical cyclone.
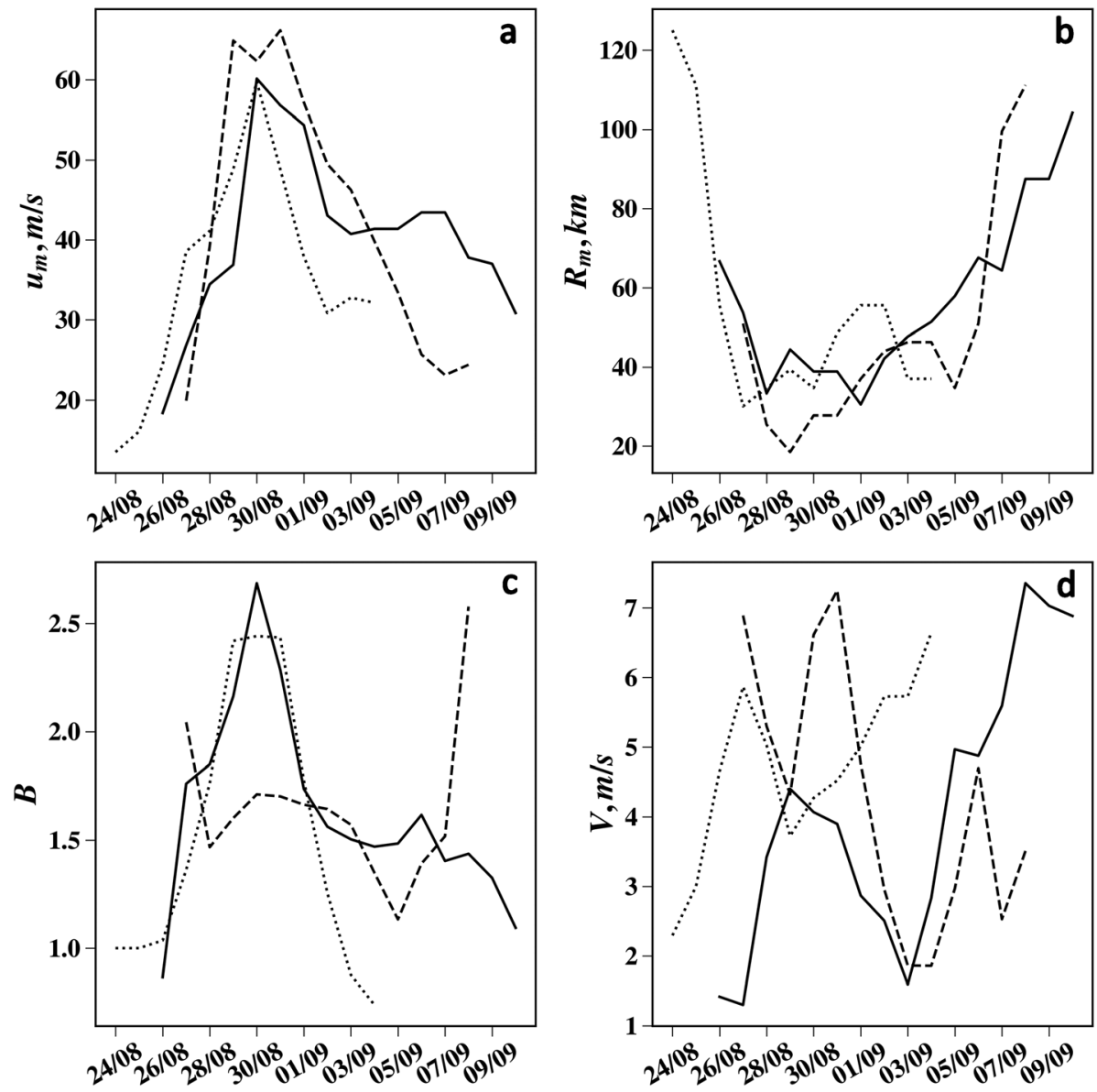

Figure 9. Parameters of TC wind field derived from BT data averaged over 1 day used in the model simulations: (a) maximum wind speed; (b) radius of max wind speed; (c) shape parameter B in model (3.16), and (d) translation velocity. Lines style: (dashed) Jimena, (dotted) Ignacio, and (solid) Kilo. BT = best-track; TC $=$ tropical cyclone. 

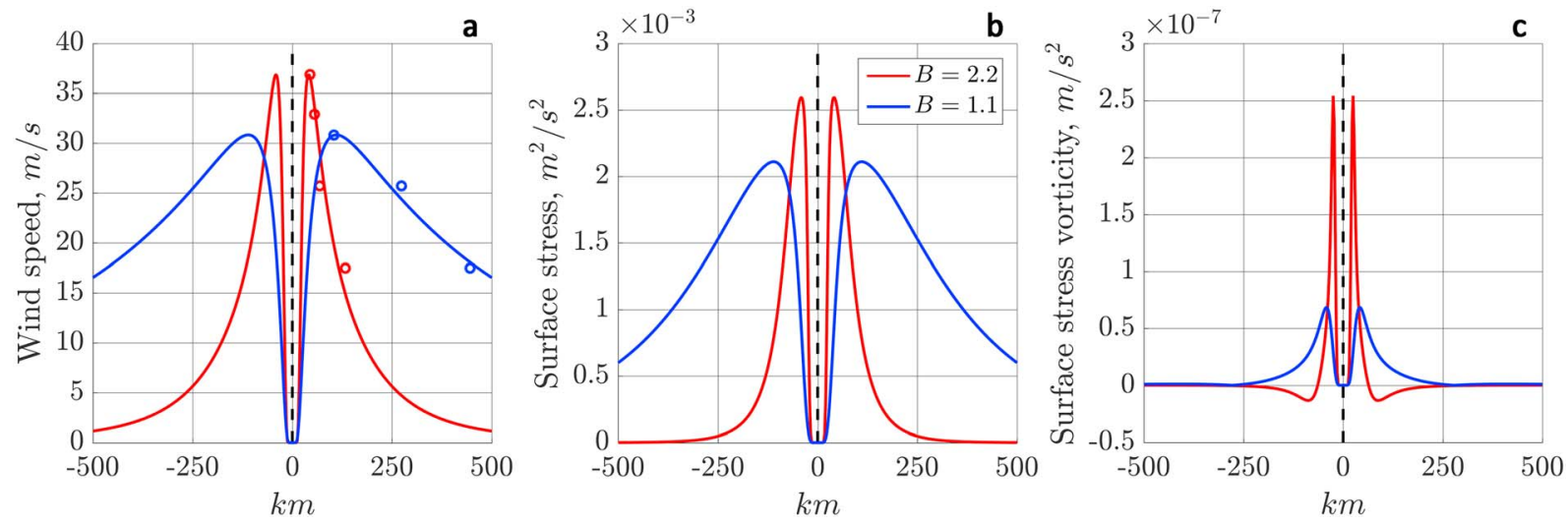

Figure 10. (a) Wind model (3.16) fitted to BT radial distribution of wind speed data shown by open circles. (b) Radial distribution of the surface wind stress corresponding to the wind speed profile shown in left plot. (c) Corresponding vorticity of the wind stress. BT $=$ best-track.

stratification. For the baroclinic responses, analytical solutions are considered, as described in section 3.1, with a three-layer approximation of the vertical stratification.

\subsection{SSH Anomalies}

Some examples for the SSH anomalies, for slow, $V<C_{1}$, and fast, $V>C_{1}$, situations are shown in Figures 11 and 12. A slow TC does not generate wave-wake, except for the case shown in Figure 11f where the wavewake results from the weak second mode. Accordingly, simulated SSH anomalies are shaped as a surface

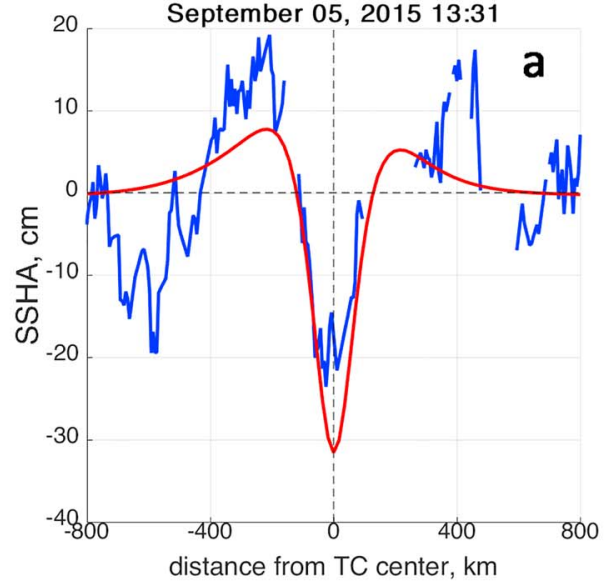

d

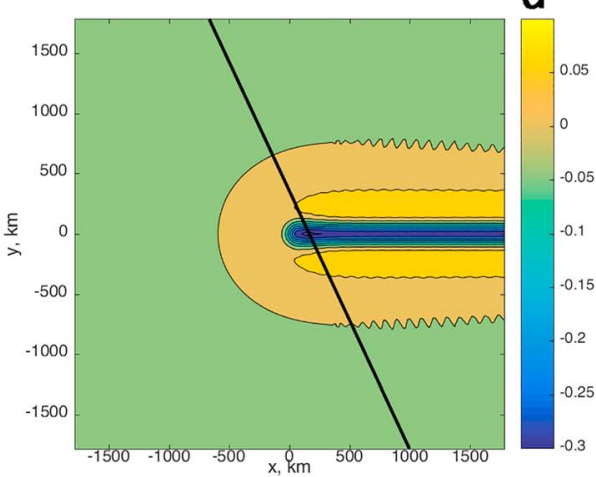

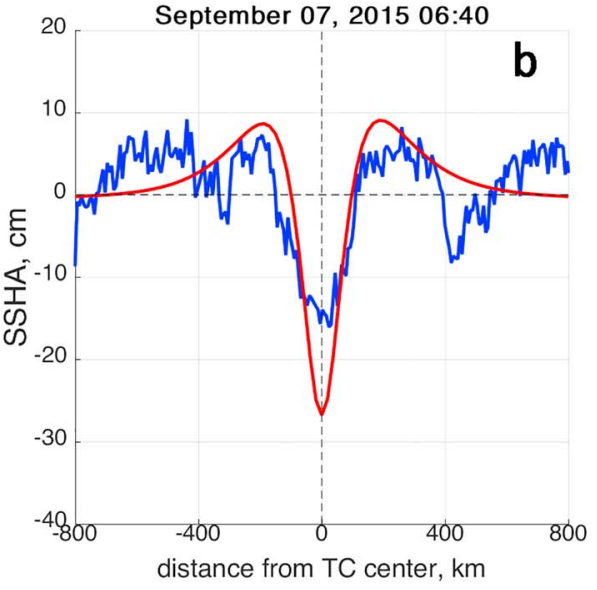

e

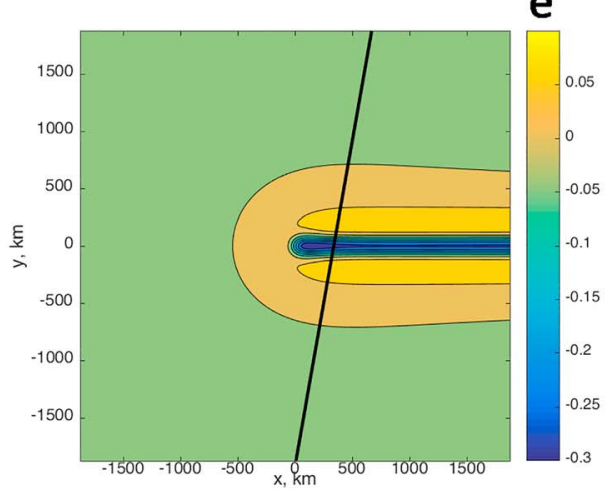

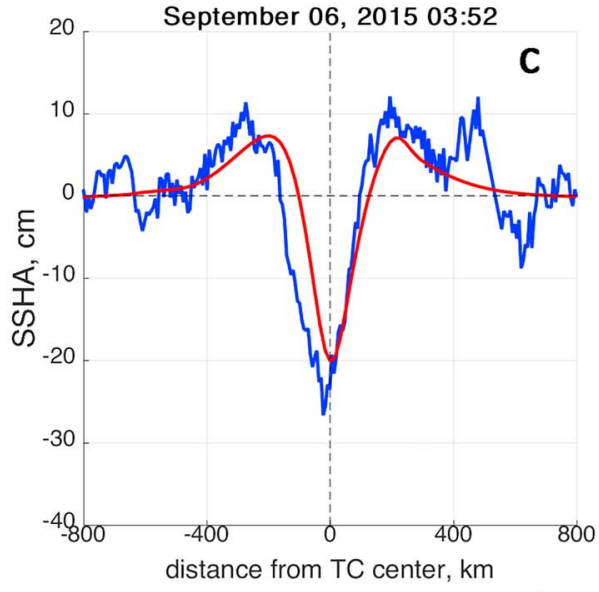

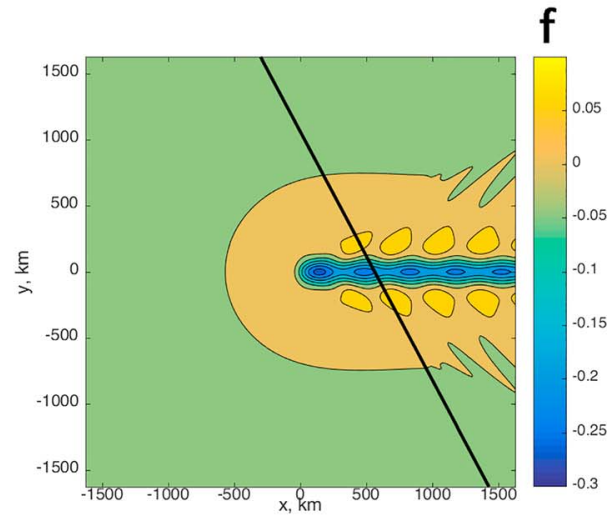

Figure 11. Cases of slow TCs. (a, b, c) Observed (blue) and simulated (red) SSH anomalies along altimeter tracks for TC Jimena (a and c) and Kilo (b). (d, e, f) Corresponding model fields of the SSH anomalies with location of altimeter tracks (shown by black line), color bars indicate SSH anomalies in meter.

Parameters of the TCs (from left to right): $V=1.9, u_{m}=46 \mathrm{~m} / \mathrm{s}, R_{m}=46 \mathrm{~km}, B=1.6 ; V=1.6 \mathrm{~m} / \mathrm{s}, u_{m}=41 \mathrm{~m} / \mathrm{s}, R_{m}=48 \mathrm{~km}, B=1.5 ; V=3 \mathrm{~m} / \mathrm{s}, u_{m}=49 \mathrm{~m} / \mathrm{s}, R_{m}=$ $44 \mathrm{~km}, B=1.6$. $\mathrm{SSH}=$ sea surface height; $\mathrm{TC}=$ tropical cyclone. 

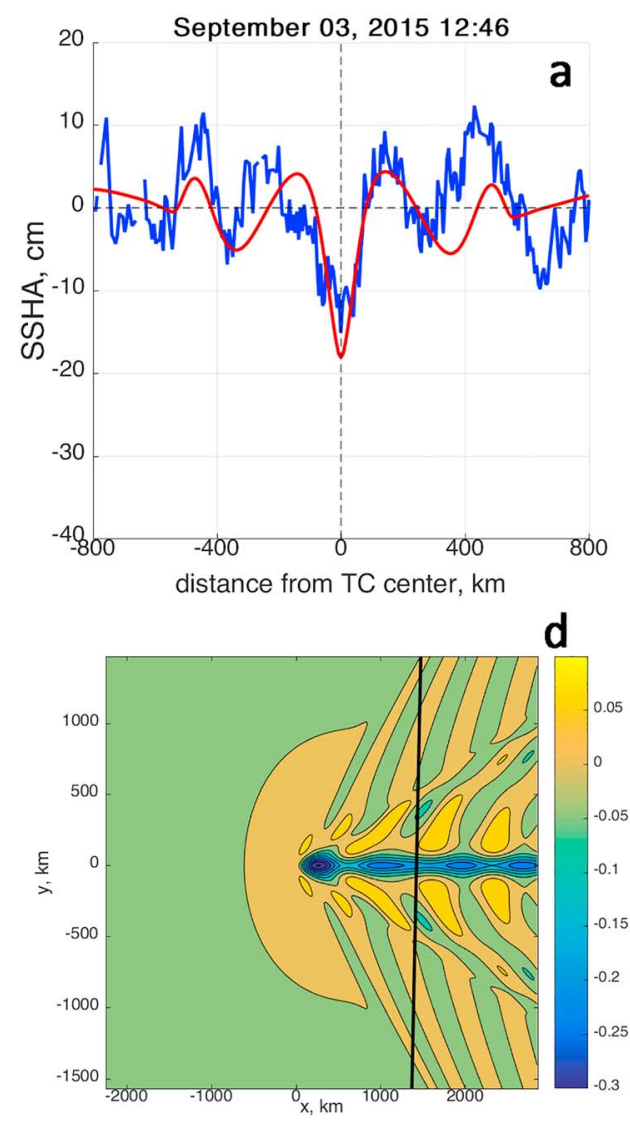
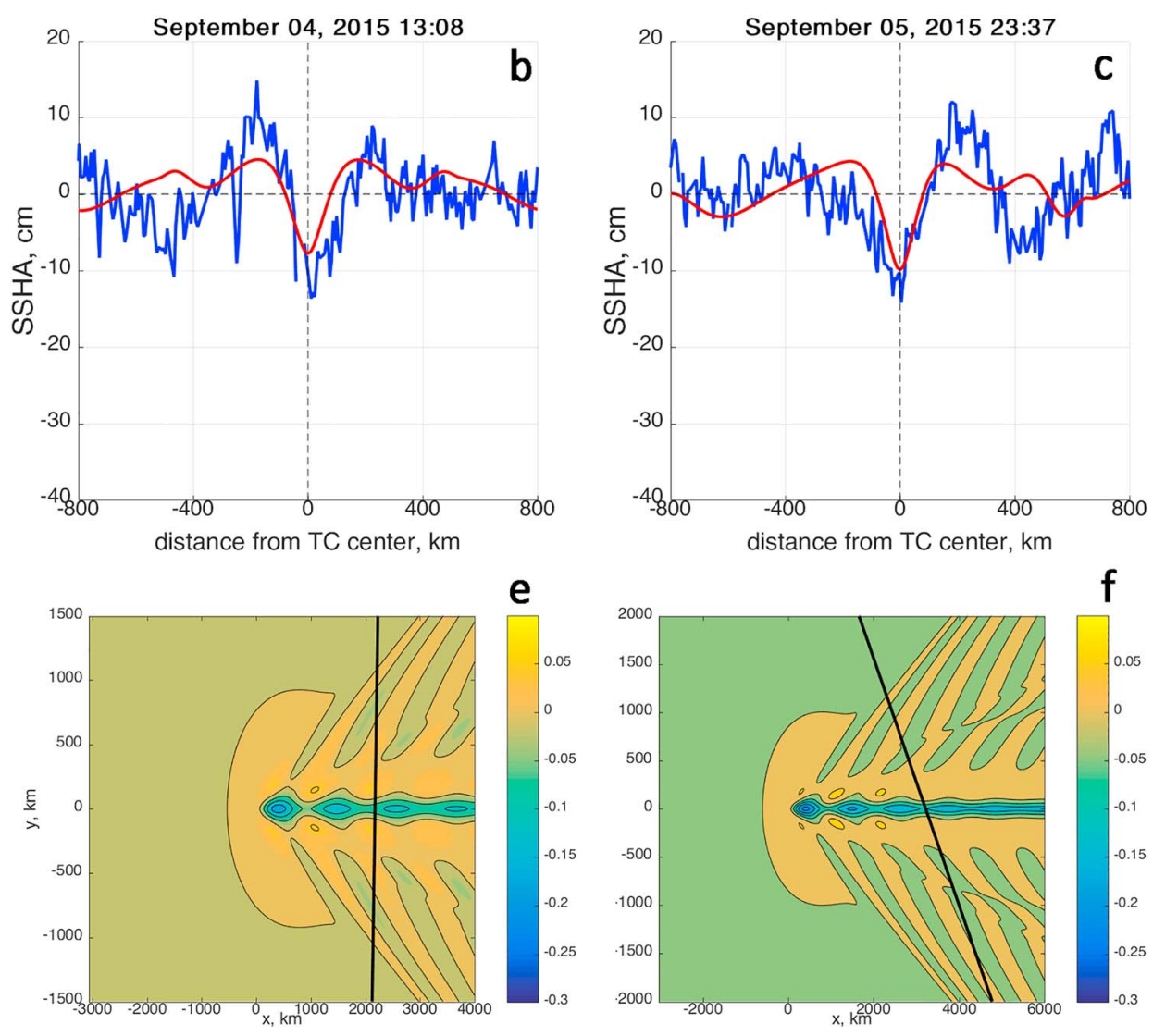

Figure 12. Cases of fast TC. (a, b, c) Observed (blue) and simulated (red) SSH anomalies along altimeters tracks for TC Jimena. (d, e, f) Corresponding model fields of the SSH anomalies with location of altimeter tracks (shown by black line), color bars indicate SSH anomalies in meter. Parameters of the TC Jimena (from left to right): $V=4.3 \mathrm{~m} / \mathrm{s}, u_{m}=65 \mathrm{~m} / \mathrm{s}, R_{m}=19 \mathrm{~km}, B=1.6 ; V=7.3 \mathrm{~m} / \mathrm{s}, u_{m}=66 \mathrm{~m} / \mathrm{s}, R_{m}=28 \mathrm{~km}, B=1.7 ; V=6.6 \mathrm{~m} / \mathrm{s}, u_{m}=6.2 \mathrm{~m} / \mathrm{s}, R_{m}=28 \mathrm{~km}, B=1.7$. $\mathrm{SSH}=$ sea surface height; $\mathrm{TC}=$ tropical cyclone.

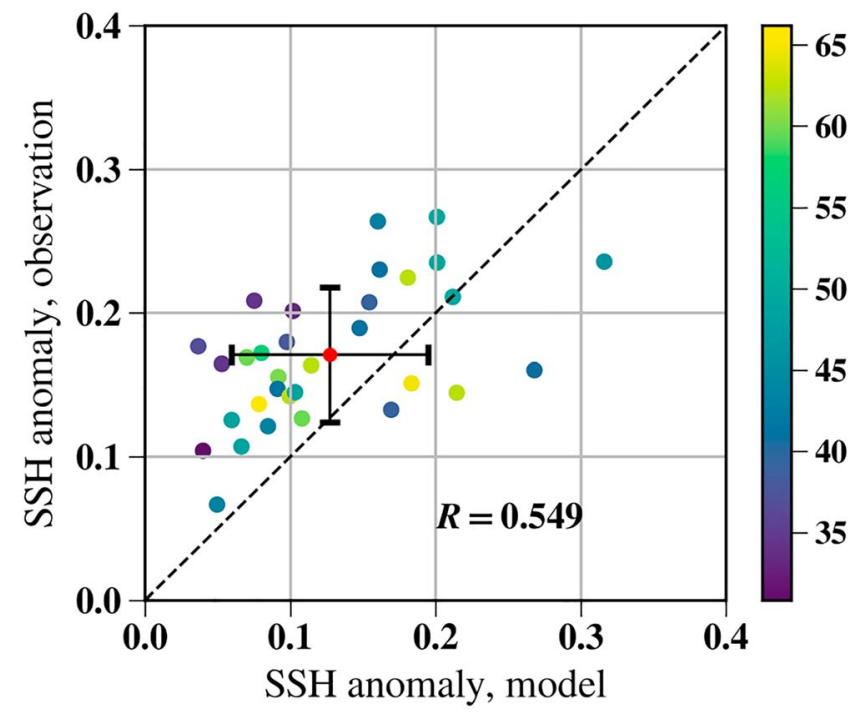

Figure 13. Scatterplot model versus observations. Color indicates wind speed, dashed line is one-to-one relation, red dot is mean value, and vertical bars are the std of the data in direction of each of the axis. SSH = sea surface height. trench, mostly generated by baroclinic modes, with a weak contribution from the barotropic mode (see also Figure 4). At variance, a fast TC generates a spectrum of IW modes, and SSH anomalies combine ocean surface undulations with dominant contribution of the two first modes, Figures $12 \mathrm{~d}$ to $12 \mathrm{f}$. Simulated transects of the SSH anomalies display marked oscillations with main troughs around the TC track. For both slow and fast situations, model estimates are consistent with observations: the slower the TC, the deeper the SSH anomalies. Overall results are summarized in Figure 13. Given its simplicity, the model results remarkably reproduce observations, with a slight overall underestimation (about 5 $\mathrm{cm})$. It should also be noticed that some remarkable differences between simulations and observations (as, e.g., in Figure 11a) result from the fact that SSH anomalies not only reflect immediate changes in the wind forcing but also anomalies associated with earlier forcing events, as well as mesoscale eddy activity. As such SSH measurements may appear contaminated by the SSH anomalies left by other TC traveled in this area before, as well as probably some other anomalies related to the mesoscale ocean dynamics.

\subsection{SST Anomalies}

SST wakes, developing behind TCs, are shown in Figure 14. Compared to observations, predicted SST wakes are generally narrower. Such a 

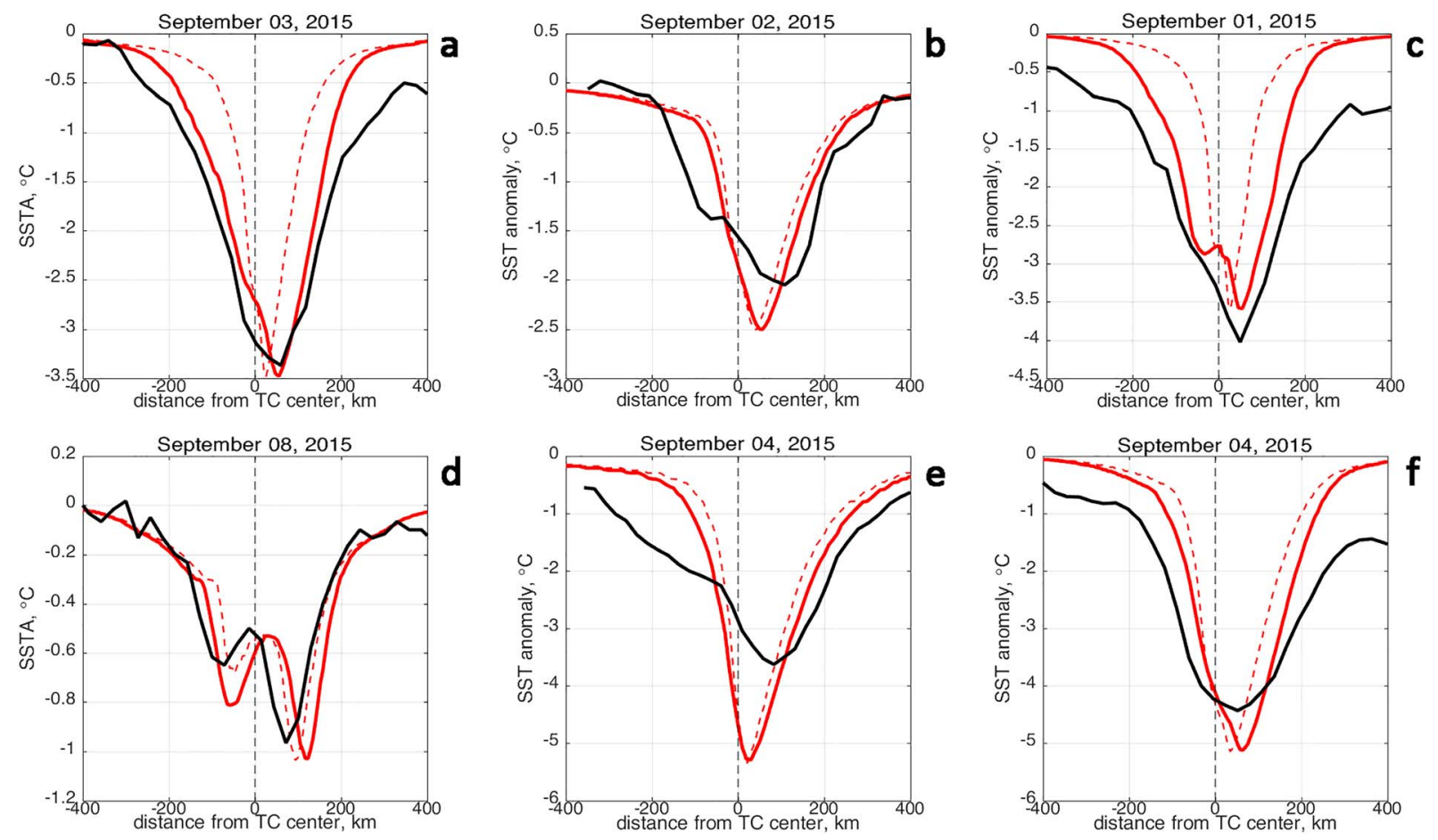

Figure 14. Comparisons of (black lines) observed and (red lines) modeled transects of the SST wakes just behind TCs (a, d) Jimena, (b, e) Ignacio, and (c, f) Kilo. Dash red lines correspond to model 28, solid red lines are model results accounting for the cross-track advection, equation (33) with 34 . SST $=$ sea surface temperature; $\mathrm{TC}=$ tropical cyclone.

discrepancy may result from a too idealized wind field description and from the broadening induced by the resolution of the satellite observations.

The role of advective transport of the SST anomalies, in the TC cross-track direction, can also be considered. The magnitude of TC-induced currents is typically much smaller than the TC translation velocity (linearized heat balance; equation (27)), but strong SST cross-track gradient may be large. To assess this contribution, equation (27) is thus modified as

$$
\partial \theta_{m} / \partial x_{1}+\left(u_{2} / V\right) \partial \theta_{m} / \partial x_{2}=-1 / 2 \bar{\Gamma} w_{e} / V
$$

where $u_{2}$ is the cross-track component of the surface current velocity in the ML, that is, $u_{2}=M_{2}^{w} / h$ with $M_{2}^{w}$ defined by equation (5) (its solution is discussed in the end of section 4.1). Equation (32) rewrites as
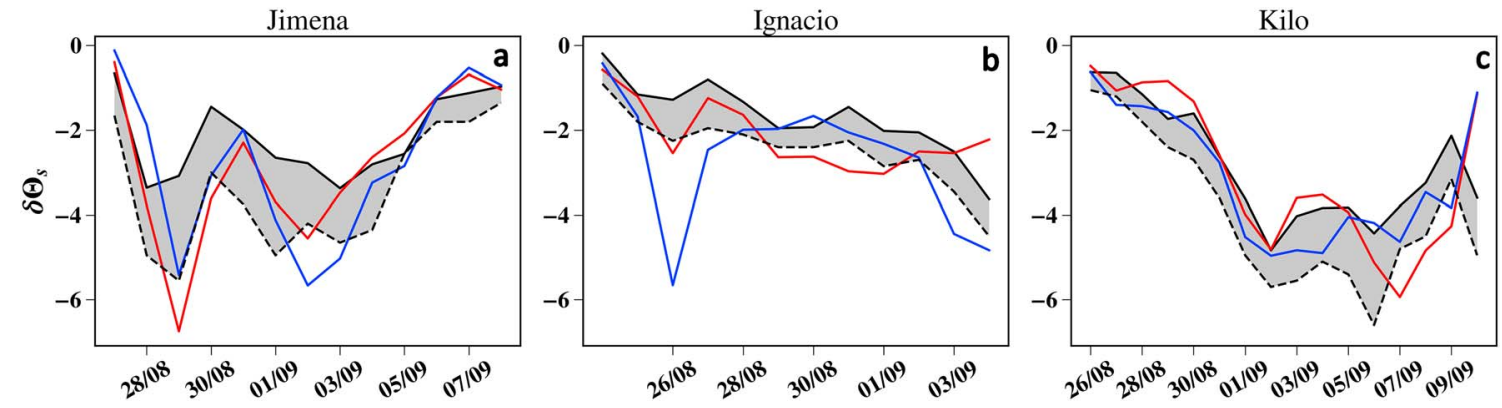

Figure 15. Along TCs track evolution of the SST anomalies for TCs (a) Jimena, (b) Ignacio, and (c) Kilo. Line style: (black solid) observed SST anomalies, (dash solid) minimal values of observed SST anomalies, (red) model simulations for WORLD OCEAN ATLAS stratification, and (blue) for ISAS-15 stratification data. $\mathrm{SST}=$ sea surface temperature; $\mathrm{TCs}=$ tropical cyclones. 


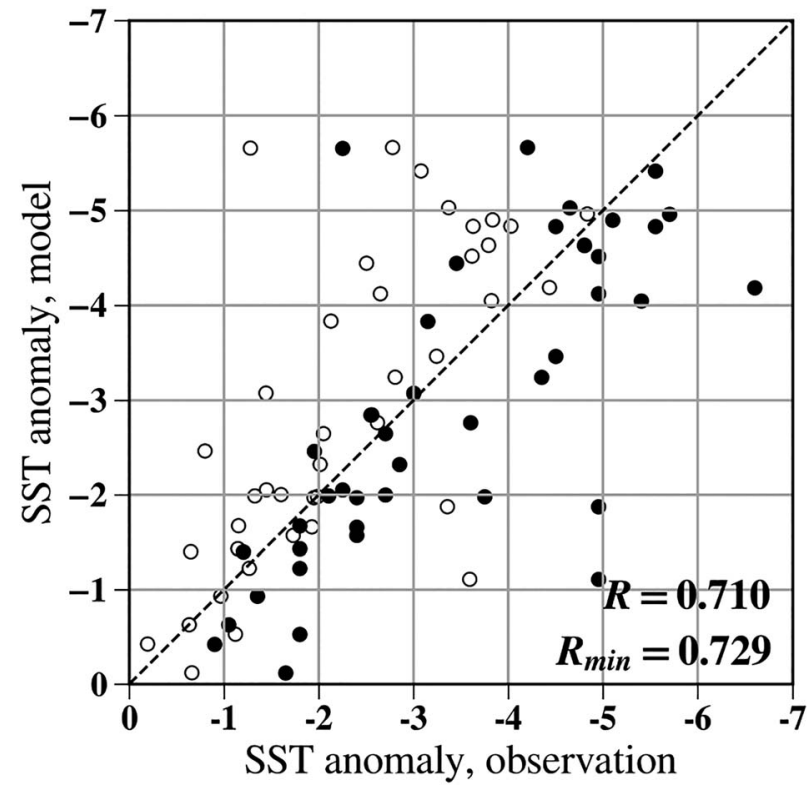

Figure 16. Observed SST anomalies versus model simulations for ISAS-15 stratification data. Dash line indicates one-to-one relation. Open circles indicate mean SST anomalies, and filled circles indicate minimal values of SST anomalies SST $=$ sea surface temperature.

$$
\mathrm{d} \theta_{m} / \mathrm{d} x_{1}=-1 / 2 \bar{\Gamma} w_{e} / V
$$

where $d / d x_{1}$ stands for total derivative along characteristics

$$
x_{2}\left(x_{1}\right)=x_{2}\left(x_{10}\right)+\int_{x_{10}}^{x_{1}}\left(u_{2} / V\right) \mathrm{d} x_{1}
$$

Since 33 is similar to 27 , the solution 28 for the SST anomalies, $\delta \theta_{s}$, should now be treated as SST field along a family of characteristics 34 . Solution of equation (33) with 34 is shown in Figure 14. The cross-track advection effectively widens the SST wake, with a stronger impact for slower TCs. This effect improves comparisons between model and observations, though some discrepancies still remain.

Figure 15 shows simulations of the along track evolution of the observed SST anomalies for the interior stratification data provided by the WORLD OCEAN ATLAS 2013 version 2, and, for comparative purposes, estimates provided by ISAS-15. Model simulations for both stratification data sets are in general very similar, justifying that shape of stratification transects provided by both data sets is similar (see Figure 8 in Kudryavtsev et al., companion paper, for ISAS-15 transects). In general, SST anomalies follows the main trend and oscillations of observed SST anomalies (both the mean and the minimal values) caused by changes of variety of the TCs parameters (wind speed, translation velocity, and radius) and environment parameters (ocean stratification and Coriolis parameter) in wide range. For TCs Kilo and Jimena, comparisons are quite remarkable, but for TC Ignacio, some deviations between the model simulations using ISAS-15 and observations around 26 August can be noticed. Simulations with stratification evaluated from the World Ocean Atlas attenuate departures between model and observations. Following ISAS-15 data, in this particular case, the TC traveled over an ocean area with the largest values for both the temperature gradients and Brunt-Väisälä frequencies
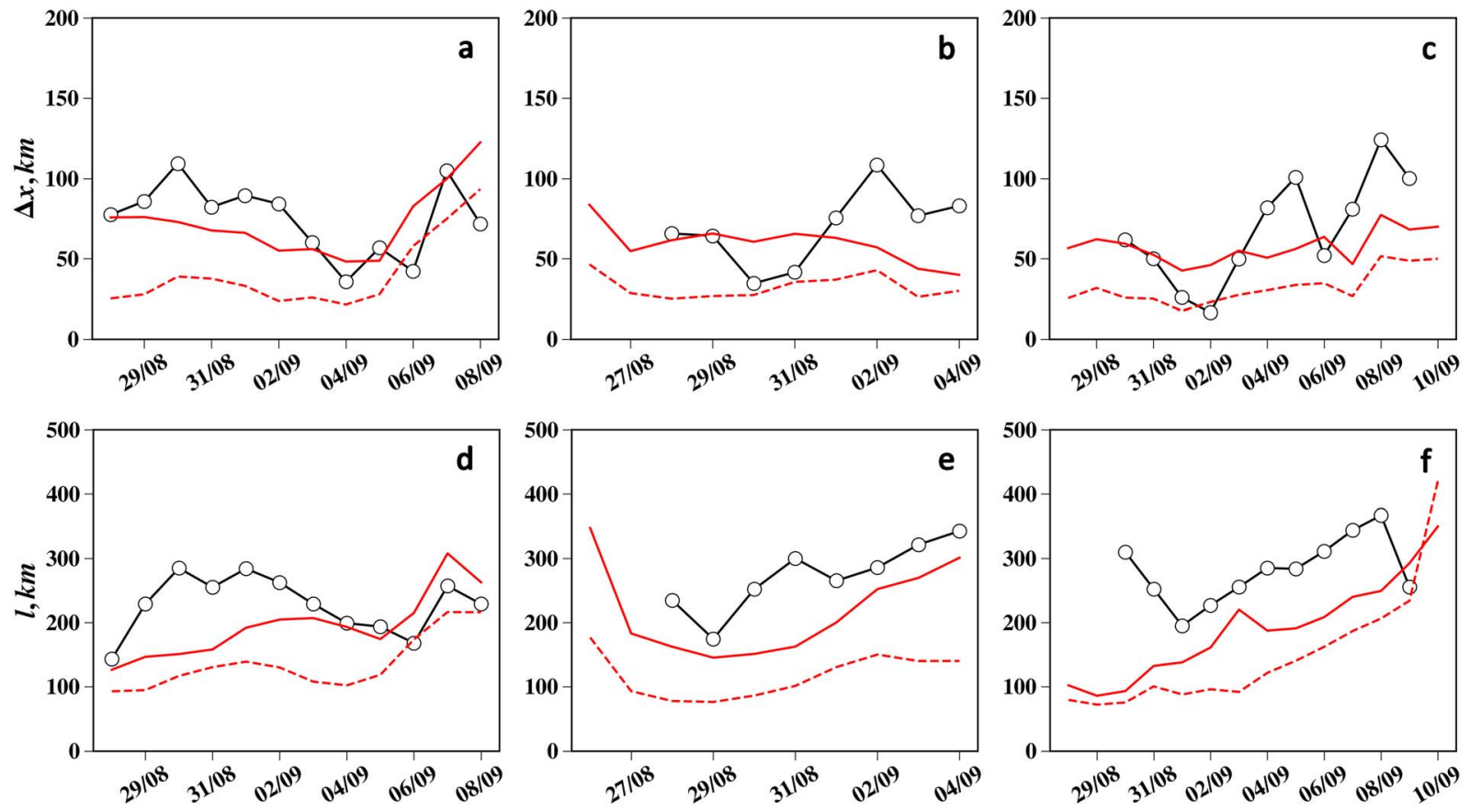

Figure 17. Along TCs track evolution of (a-c) offset and (d-f) width of the SST anomalies for TCs (a, d) Jimena, (b, e) Ignacio, and (c, f) Kilo. Line style: (black line with open circles) observations and (red dashed and red solid) model simulations without (equation 28) and with (equation 33 with 34 ) cross-track advection by wind-driven current, respectively. SST $=$ sea surface temperature; $\mathrm{TCs}=$ tropical cyclones. 

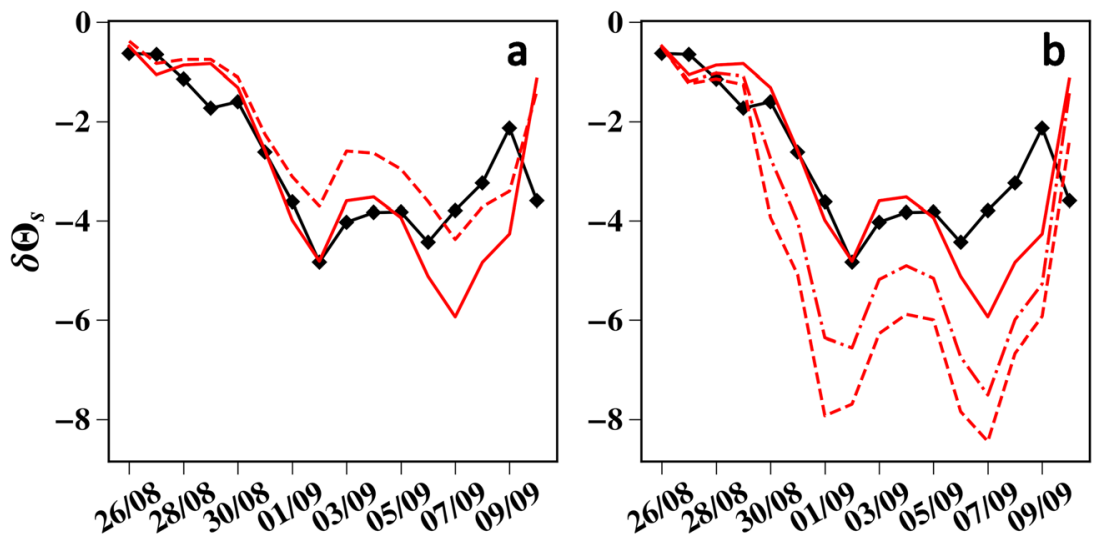

Figure 18. Along-track evolution of SST anomalies for TC Kilo; observations are shown by black lines with dots. (a) Full model (red solid) and model without accounting for the upwelling (red dashed). (b) Model simulations with different drag coefficients: (red solid) suggested $C_{d}$, (red dashed) $C_{d}$ corresponding to the Charnock relation extrapolated to high winds, (red dash-dotted), and $C_{d}$ corresponding to the Charnock relation but saturated at $C_{d}=2 \times 10^{-3}$. SST $=$ sea surface temperature; $\mathrm{TC}=$ tropical cyclone.

in the seasonal pycnocline, see Figures 8 and 10 from Kudryavtsev et al., companion paper. According to the ISAS-15 data (see Figure 8 from the companion paper), these regions are also located in the vicinity of the ocean surface frontal zone. Actual bias of this frontal zone from an observed point may then lead to significant deviation of actual stratification from ISAS-15 data and thus to lower model estimates of the SST anomaly.

Scatterplot, Figure 16, exhibits quantitative correspondence of the model to observations with rather high level of correlation, especially for the model correlation with minimum of the SST in the wake.

Model simulations of the TC-track offsets and widths of the SST anomalies underestimate the observation, Figure 17. Considering cross-track advection, solutions 33 with 34, improves the model performances. However, the cold wake width is still largely underestimated. It may be speculated that more realistic TC wind fields, with possible marked asymmetries between left and right sectors, could improve the comparisons.

\subsection{Influence of Drag Coefficient Parametrization}

Given the proposed model simplicity, we can readily explore the idea that SST and SSH surface expressions, along with knowledge of the initial conditions, can be used to test and possibly infer the drag coefficient relationship with wind speed. Already, the suggested parametrization is consistent with reported observations (Powell et al., 2003) and altimeter-derived SSH anomalies, see equation (18) and Figure 15 from Kudryavtsev et al., companion paper. The present model can as well be used to test the sensitivity to drag coefficient, $C_{d}$, alternatively defined as (a) $C_{d}$ prescribed by the Charnock relation extrapolated to high winds, $C_{d 1}$; and (b) $C_{d}$ saturating at a given threshold value, for example, $C_{d}^{\text {st }}=2 \times 10^{-3}$, that is, $C_{d 2}=\min$ $\left(C_{d 1}, C_{d}^{\mathrm{st}}\right)$. Results are reported in Figure $18 \mathrm{~b}$. As expected, model simulations using different drag coefficients result in larger SST anomalies, largely overestimating the observations. This is consistent with simulations reported by Sanford et al. (2007).

Similar effects apply to SSH anomalies; two examples for slow and fast TCs are shown in Figure 19. While more investigations are certainly needed, observations and simulations highlight the potential to combine both SST and SSH wake signatures to provide necessary information to improve understandings and quantify momentum exchanges at the sea surface under extreme conditions. As presented, the present results suggest a relative saturation of the surface stress, that is, reduction of the drag coefficient, to bring model estimates in reasonable agreement with observations.

To note, the present model setup can also be used to test impacts of asymmetrical wave fields, that is, associated with possible trapping and enhancement of large surface gravity waves in the front-right storm quadrant (Kudryavtsev et al., 2015; Young, 2006). Indeed, wave-induced mixing, including breaking impacts and 

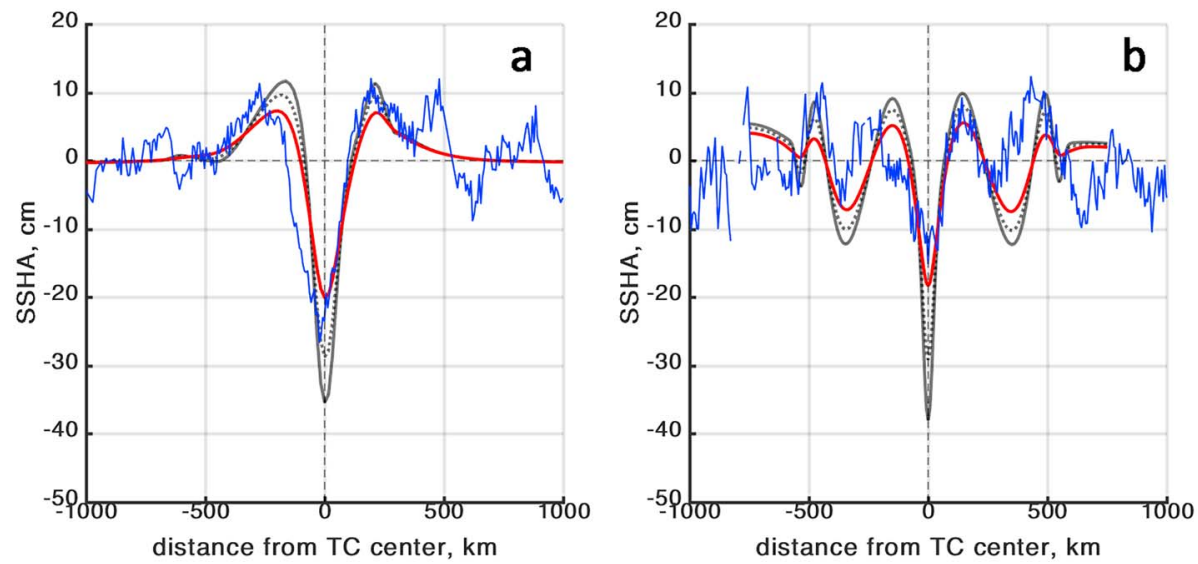

Figure 19. Examples of (blue lines) observations and model simulations of the SSH anomalies for (a) slow and (b) fast TC, shown before in Figures 11 and 12. Red lines are model simulations with basic drag coefficient, solid and dotted black lines are model simulations with $C_{d}$ defined by the Charnock relation extrapolated to high winds and $C_{d}$ defined by the Charnock relation but saturated at $C_{d}=2 \times 10^{-3}$ correspondingly. $\mathrm{SSH}=$ sea surface height; TC $=$ tropical cyclone

the combined action of wave-induced drift and wind-induced shear (e.g., Reichl et al., 2016), certainly contributes to the turbulent kinetic energy (TKE) balance equation. Yet, within the TC core, the expected ML deepening, equation (11), may largely exceed the depth strongly influenced by wave breaking and nonbreaking waves that is of the order $d_{w} \approx 1 /\left(3 k_{p}\right)$, with $k_{p}$ the spectral peak wave number (Babanin \& Haus, 2009; Kudryavtsev et al., 2008). In that case, wave-induced mixing should not impact the SST anomalies induced by TC, as recently reported by Stoney et al. (2017). Moreover, the present results suggest a relative saturation of the surface stress. As such, while existing, local particular influences of surface waves and asymmetrical wave fields may not be strongly distinguishable.

On the other hand, nonlocal surface wave impacts may well be considered. Indeed, as discussed in paragraph 4.2, SST anomalies are very sensitive to the vertical distribution of the prestorm temperature. Fore-runner waves, traveling with group velocities largely exceeding the TC-translation velocity, will disturb (a priori) quite upper ocean areas. The prestorm stratification shall then be modified, before the TC-core arrival time. This enhanced nonbreaking wave-induced mixing process is thus nonlocal. The depth, $d_{w} \approx 1 /\left(3 k_{p}\right)$, influenced by surface waves (Babanin \& Haus, 2009) shall thus be related to TC characteristics at previous time steps. Such a nonlocal impact can then be suggested to enter the correcting factor $\left(1-h_{0}^{2} / h^{2}\right)$, with a thickness, $h_{0}=d_{w}$, now more directly related to wave parameters, for example, peak frequency/wave number (see, e.g., equations (6) and (15) for trapped waves in Kudryavtsev et al., 2015).

\section{Conclusion}

In this paper, the previously proposed framework, used to help interpret satellite observations (Kudryavtsev et al., companion paper), is extended to provide a more complete analytical description of ocean responses to moving TC. As developed, the proposed model helps detail the wind-driven current field, the upper ML cooling and its evolution, with the associated space-time variability of the pycnocline caused by TC-induced baroclinic motions. While more complete, the goal, and main purpose of the present paper, is to best bypass the computational burden of advanced numerical simulations. Simplified analytical solutions are thus elaborated. As tested, the derived solutions are very fast to compute, and help to assess, on quantitatively correct levels, the sensitivity of ocean responses to moving TCs, specified by various environmental (wind forcing and ocean stratification) conditions.

To go beyond classical 1D ML models, the suggested solutions build on previously developed linear models (Geisler, 1970; Orlanski \& Polinsky, 1983) of ocean response to transient events. The final model is then represented by a set of analytical solutions, describing baroclinic and barotropic responses, and resulting SST anomalies. The baroclinic response is decoupled from the ML, in that sense that disturbances of the 
upper ocean stratification caused by the ML development do not strongly impact dynamics of baroclinic modes. This assumption is accurate to the smallness of the parameter $1 / 2\left(N_{1} h / C\right)^{2}$ utilizing Brunt-Väisälä frequency in the seasonal pycnocline, the ML depth, and phase velocity of long internal waves. Accordingly, the baroclinic response can be estimated for prestorm ocean stratification. Simple analytical solutions are then derived by fitting the prestorm stratification with a three-layer description, to take into account seasonal and main thermocline, and abyssal characteristics. To the contrary, the ML is strongly coupled with these interior motions, through the TC-induced upwelling effect that affects the entrainment velocity 26 . The resulting ML temperature is further strongly dependent on the integrated temperature gradient in the upper layer, equation (27) with 7.

Comparisons with observations proved the model capable to reproduce observed shape and magnitudes of the SSH and SST anomalies on quantitative levels. Supporting Yablonsky and Ginis (2009), the model simulations provide solid evidences about the key role of TC-induced upwelling, caused by vertical baroclinic motions, to control the ML cooling, see Figure 18a. This is especially effective for slowly translating TCs. Cross-track advection by wind-driven currents, though small compared with TC translation velocity, further remarkably contributes to broaden the shape of the SST wake, for example, Figure 17. These quantitative agreements are obtained for various TC characteristics, ocean stratification conditions, and Coriolis parameters. It is worthy to note that the proposed model does not possess special/dedicated tuning constants, besides the critical Richardson number, equation (8). For the simulations, it is here set as $R_{\mathrm{cr}}=0.6$, following previous studies (Price, 1981). Yet, the ML depth (11) solely depends on this critical $R_{\mathrm{cr}}$ in power one fourth, and changing this tuning parameter shall not impact the results significantly.

Given the proposed model simplicity, we further test the idea that SST and SSH surface expressions, along with knowledge of the initial conditions, can be used to test and possibly infer the drag coefficient relationship with wind speed. As presented, the obtained results suggest a relative saturation of the surface stress under extreme conditions, that is, reduction of the drag coefficient, bringing model estimates in reasonable agreement with observations. Since a nearly saturated surface stress may occur under high winds, it can be speculated that peculiarities of the resulting wave field may not particularly impact the upper ocean response within the TC-core area. More plausible, outrunning energetic surface waves, related to TC characteristics at previous time steps, may have important nonlocal contributions. As precursors to the forthcoming TC intense forcing, surface waves can destabilize the preexisting upper layer stratification. As such, surface waves may effectively, but through a so-called nonlocal effect, contribute to modulate the intensity and extent of TC-cooling wakes.

More investigations are certainly needed, but observations and simulations already highlight the potential to combine both SST and SSH wake signatures. In that context, analysis can certainly already build on the present-day altimeter constellation: up to six satellite altimeters are today available. It can further be anticipated that next National Aeronautics and Space Administration's Surface Water and Ocean Topography mission (Fu et al., 2012), with unprecedented 2D altimeter mapping capabilities, promises to greatly improve the analysis of TC-induced SSH wake: this shall be highly beneficial to improve our knowledge of the air-sea exchanges under extreme conditions.

Finally, given the complexity of isolating the governing physical processes in full-physics coupled models, the proposed developments can be introduced as a computational module into an atmospheric numerical model of TC evolution coupled with the ocean through the resulting surface enthalpy fluxes (e.g., Yablonsky et al., 2015). Furthermore, given its effectiveness and low computational burden, the model can be rapidly evaluated under a wide range of possible initial conditions, to best sample the whole uncertainty space to apply inverse approaches (e.g., Sraj et al., 2013).

\section{Appendix A}

\section{A1. Main Equations}

Solutions of equation (13) for each layer of the three-layer stratification description, satisfying dynamic and kinematic boundary conditions, read 


$$
\begin{gathered}
\widehat{w}_{1}\left(x_{3}\right) / \widehat{w}_{h}=\cos \left[N_{1}\left(x_{3}+h\right) / c\right]+\frac{\cos (\Sigma+\varphi)-n \cos (\delta-\varphi)}{\sin (\Sigma+\varphi)-n \sin (\delta-\varphi)} \sin \left[N_{1}\left(x_{3}+h\right) / c\right] \\
\widehat{w}_{2}\left(x_{3}\right) / \widehat{w}_{h}=\frac{2 N_{1} /\left(N_{1}+N_{2}\right)}{\sin (\Sigma+\varphi)-n \sin (\delta-\varphi)} \sin \left[N_{2}\left(D+x_{3}\right) / c+\varphi\right] \\
\widehat{w}_{3}\left(x_{3}\right) / \widehat{w}_{2}(-D)=\frac{H+x_{3}}{H-D}
\end{gathered}
$$

where

$$
\widehat{w}_{h}=-\frac{\widehat{F}}{\Omega^{2}-f^{2}}\left[1+\left(N_{1} h / c\right) \frac{\cos (\Sigma+\varphi)-n \cos (\delta-\varphi)}{\sin (\Sigma+\varphi)-n \sin (\delta-\varphi)}+\frac{1}{2}\left(N_{1} h / c\right)^{2}\right]^{-1}
$$

is the vertical velocity at the ML base, $c=\sqrt{\Omega^{2}-f^{2}} / k$ is a variable with dimension of a velocity, $n=\left(N_{1}-N_{2}\right) /\left(N_{1}+N_{2}\right)$, and

$$
\begin{aligned}
& \Sigma=N_{1}(d-h) / c+N_{2}(D-d) / c \\
& \delta=N_{1}(d-h) / c-N_{2}(D-d) / c \\
& \sin \varphi=\frac{N_{2}(H-D) / c}{\sqrt{1+\left[N_{2}(H-D) / c\right]^{2}}}
\end{aligned}
$$

To derive A.4, $g \Delta \rho / \rho$ is evaluated as $g \Delta \rho / \rho=-N_{1}^{2} h / 2$.

In the open ocean conditions, typical values of $N_{1}$ are of order $N_{1} \propto 10^{-2} 1 / \mathrm{s}$ or less, and the ML depth is of order $h \propto 10$ to $10^{2} \mathrm{~m}$. If $c$ corresponds to the phase velocity of long IWs (which is about $c \approx 3 \mathrm{~m} / \mathrm{s}$ ), then the magnitude of the parameter $N_{1} h / c$ is in the range $N_{1} h / c=3 \times\left(10^{-2}\right.$ to $\left.10^{-1}\right)$, that is, can be considered small. To the smallness accuracy of the term $1 / 2\left(N_{1} h / c\right)^{2} \ll 1$, relationships A1 and A.2 can be rewritten as

$$
\begin{gathered}
\widehat{w}_{1}\left(x_{3}\right) / \widehat{w}_{0}=\cos \left[N_{1} x_{3} / c\right]+\frac{\cos \left(\Sigma_{0}+\varphi\right)-n \cos \left(\delta_{0}-\varphi\right)}{\Delta} \sin \left[N_{1} x_{3} / c\right] \\
\widehat{w}_{2}\left(x_{3}\right) / \widehat{w}_{0}=\frac{2 N_{1} /\left(N_{1}+N_{2}\right)}{\Delta} \sin \left[N_{2}\left(D+x_{3}\right) / c+\varphi\right]
\end{gathered}
$$

where

$$
\widehat{w}_{0}=-\frac{\widehat{F}}{\Omega^{2}-f^{2}}
$$

and

$$
\begin{aligned}
& \Sigma_{0}=N_{1} d / c+N_{2}(D-d) / c \\
& \delta_{0}=N_{1} d / c-N_{2}(D-d) / c \\
& \Delta=\sin \left(\Sigma_{0}+\varphi\right)-n \sin \left(\delta_{0}-\varphi\right)
\end{aligned}
$$

These relations do not explicitly depend on either $h$ or $g \Delta \rho / \rho$, demonstrating that for the considered conditions, the ML does not impact the baroclinic motions in the interior layer.

\section{A2. Solutions}

Relations (A.6) and (A.7) display a singularity in the vicinity of $C_{n}$, subsequent solution of equation

$$
\Delta \equiv \sin \left[\frac{N_{1} d}{C_{n}}+\frac{N_{2}(D-d)}{C_{n}}+\varphi\right]-\frac{N_{1}-N_{2}}{N_{1}+N_{2}} \sin \left[\frac{N_{1} d}{C_{n}}-\frac{N_{2}(D-d)}{C_{n}}-\varphi\right]=0
$$

Solution of this equation defines the phase velocity of IWs, $C_{n}$, for the different modes, $n$, for given parameters of the ocean stratification. 
Once equation (A.10) is solved and the phase velocity of IW defined, denominator in A.6 and A.7 can be expanded to the first order of $\left(\Omega-\Omega_{0}\right)$, that is, around the resonance curve $\Omega_{0}=\sqrt{f^{2}+k^{2} C_{n}^{2}}$ :

$$
\begin{gathered}
\Delta(\Omega)=\frac{\partial \Delta \partial C_{n}}{\partial C_{n} \partial \Omega}\left(\Omega-\Omega_{0}\right) \\
=-\frac{1}{2\left(\Omega^{2}-f^{2}\right)}\left[\Sigma_{0} \cos \left(\Sigma_{0}+\varphi_{n}\right)-n \delta_{0} \cos \left(\delta_{0}-\varphi_{n}\right)\right]\left(\Omega^{2}-\Omega_{0}^{2}\right)
\end{gathered}
$$

In this case, expressions (A.6) and (A.7) for the vertical velocity read

$$
w_{n}(\boldsymbol{x}, z)= \begin{cases}a_{1 n} W_{n}(\boldsymbol{x}) \sin \left[N_{1} x_{3} / C_{n}\right], & \text { at }-d<x_{3}<0 \\ a_{2 n} W_{n}(\boldsymbol{x}) \sin \left[N_{2}\left(D+x_{3}\right) / C_{n}+\varphi_{n}\right], & \text { at }-D<x_{3}<-d\end{cases}
$$

where $a_{1 n}$ and $a_{2 n}$ are dimensionless vertical velocity amplitudes depending on stratification as

$$
\begin{aligned}
& a_{1 n}=-\frac{2 C_{n}}{\Sigma_{0}}\left[\frac{\cos \left(\Sigma_{0}+\varphi_{n}\right)-n \cos \left(\delta_{0}-\varphi_{n}\right)}{\cos \left(\Sigma_{0}+\varphi\right)-n\left(\delta_{0} / \Sigma_{0}\right) \cos \left(\delta_{0}-\varphi\right)}\right] \\
& a_{2 n}=-\frac{2 C_{n}}{\Sigma_{0}}\left[\frac{2 N_{1} /\left(N_{1}+N_{2}\right)}{\cos \left(\Sigma_{0}+\varphi_{n}\right)-n\left(\delta_{0} / \Sigma_{0}\right) \cos \left(\delta_{0}-\varphi_{n}\right)}\right]
\end{aligned}
$$

Parameters $\Sigma_{0}$ and $\delta_{0}$ are given by A.9 with $c$ equal to IW phase velocity, $C_{n}$, and $W_{n}(\boldsymbol{x})$ is a function defining 2D field of vertical velocity for $n$ mode, which in Fourier space reads

$$
\widehat{W}_{n}\left(k_{1}, k_{2}\right)=-\frac{\widehat{F}\left(k_{1}, k_{2}\right)}{\Omega^{2}-\Omega_{0}^{2}}=-\frac{\widehat{F}\left(k_{1}, k_{2}\right)}{C_{n}^{2}}\left[\frac{1}{k_{1}^{2}\left(V^{2} / C_{n}^{2}-1\right)-\kappa^{2}-k_{2}^{2}}\right]
$$

where $\kappa=f / C_{n}$ is the inverse baroclinic radius of deformation. Expression (A.14) corresponds to the classical equation for vertical velocity in a two-layer model with constant density at each of the layer (Geisler, 1970, his equation (17)). Inverse Fourier transformation of A.14 gives the vertical velocity in physical space that appeared in A.12

$$
W_{n}\left(x_{1}, x_{2}\right)=-\frac{1}{4 \pi^{2} C_{n}^{2}} \iint \frac{\widehat{F}\left(k_{1}, k_{2}\right)}{\left(V^{2} / C_{n}^{2}-1\right) k_{1}^{2}-k_{2}^{2}-\kappa^{2}} \exp \left(i k_{\beta} x_{\beta}\right) \mathrm{d} k_{1} \mathrm{~d} k_{2}
$$

If the translation velocity of TC is fast enough and satisfies the condition $V>C_{n}$, then A.15 has a singularity around the resonant curve in the wave number space

$$
k_{1}^{2}\left(V^{2} / C_{n}^{2}-1\right)-k_{2}^{2}-\kappa^{2}=0
$$

Analytical solution for this case is given by Geisler (1970, his equation (30)), which can be adopted. However, we suggest a slightly different form that can easily be implemented for numerical calculations using fast Fourier transform.

To simplify A.15, we suppose the main contribution to integral in A.15 to arise in the vicinity of a resonant curve in the wave number space:

$$
\begin{aligned}
& k_{1}= \pm k_{10} \\
& k_{10}=\sqrt{\left(k_{2}^{2}+\kappa^{2}\right) /\left(V^{2} / C_{n}^{2}-1\right)}
\end{aligned}
$$

After substitution of $k_{1}= \pm k_{10}+\Delta k_{1}$ in A.15 and accounting for

$$
\int\left[\exp \left(i \Delta k_{1} x_{1}\right) / \Delta k_{1}\right] \mathrm{d} \Delta k_{1}=i \pi
$$

we arrive at 


$$
W\left(x_{1}, x_{2}\right)=-\frac{i}{4 \pi\left(V^{2}-C_{n}^{2}\right)} \int\left(\frac{\widehat{F}\left(k_{01}, k_{2}\right) e^{i k_{01} x_{1}}}{k_{01}}\right) e^{i k_{2} x_{2}} \mathrm{~d} k_{2}
$$

Using by definition

$$
\widehat{F}\left(k_{01}, k_{2}\right)=\int \widehat{F}\left(x_{1}, k_{2}\right) e^{-i k_{01} x_{1}^{\prime}} \mathrm{d} x_{1}^{\prime}
$$

and introducing the radiation conditions (Lighthill, 1967), stating in our case (TC moves in direction opposite to the $-x$ axis) that disturbances generated by a point source located at $x_{1}=x_{1}^{\prime}$ can exclusively be observed at $x_{1}>x_{1}^{\prime}$, we finally get

$$
W\left(x_{1}, x_{2}\right)=\frac{1}{2 \pi\left(V^{2}-C_{n}^{2}\right)} \int_{-\infty}^{x} \mathrm{~d} x_{1}^{\prime} \int_{-\infty}^{\infty} \widehat{F}\left(x_{1}^{\prime}, k_{2}\right) \frac{\sin \left(k_{01}\left(x_{1}-x_{1}^{\prime}\right)\right)}{k_{01}} e^{i k_{2} x_{2}} \mathrm{~d} k_{2}
$$

where $\widehat{F}\left(x_{1}^{\prime}, k_{2}\right)$ is $k_{2}$ Fourier transform of wind stress source for given $x_{1}^{\prime}$, and the factor 2 is introduced to preserve the total energy of the source. Only the real part of A.21 must be taken into account.

If the TC is slow, that is, $V<C_{n}$, then relation (A.15) is not singular, and hence, the vertical velocity can be found directly as Fourier transform of A.15.

Solutions A.21 for fast TC, $V>C_{n}$, and A.15 for slow TC, $V<C_{n}$, provide, together with A.10 and A.12, the full description of baroclinic vertical motions caused by the TC.

\section{Acknowledgments}

The core support for this work was provided by the Russian Science Foundation through the Project 17-7730019 at Russian State

Hydrometeorological University. The support from the Ministry of Science and Education (Goszadanie $5.2928 .2017 / \mathrm{PP}$ ) is also acknowledged. This study was also conducted within the Ocean Surface Topography Science Team (OSTST) activities. OSTST is led by CNES and NASA, and a grant was awarded by the TOSCA board to the SILLAGE project in the framework of the CNES/EUMETSAT Research Announcement CNES-DSP/OT 122118. The data used in this paper are available at http://data.remss.com/sst/ daily_v04.0/mw/2015/; http://www. nodc.noaa.gov/; https://earth.esa.int/; http://www.aviso.altimetry.fr/; http:// ftp.nhc.noaa.gov/atcf/, and https:// www.nodc.noaa.gov/OC5/woa13/.

\section{References:}

Babanin, A. V., \& Haus, B. K. (2009). On the existence of water turbulence induced by nonbreaking surface waves. Journal of Physical Oceanography, 39(10), 2675-2679. https://doi.org/10.1175/2009JPO4202.1

Balaguru, K., Chang, P., Saravanan, R., Leung, L. R., Xu, Z., Li, M., \& Hsieh, J. S. (2012). Ocean barrier layers' effect on tropical cyclone intensification. Proceedings of the National Academy of Sciences, 109(36), 14,343-14,347. https://doi.org/10.1073/ pnas. 1201364109

Cione, J. J., \& Uhlhorn, E. W. (2003). Sea surface temperature variability in hurricanes: Implications with respect to intensity change. Monthly Weather Review, 131(8), 1783-1796. https://doi.org/10.1175//2562.1

Fu, L. L., Alsdorf, D., Morrow, R., Rodriguez, E., \& Mognard, N. (2012). SWOT: The Surface Water and Ocean Topography Mission: Wideswath altimetric elevation on Earth. Tech. Rep. Publ. 12-05, Jet Propulsion Lab, Pasadena, CA.

Geisler, J. E. (1970). Linear theory of the response of a two layer ocean to a moving hurricane. Geophysical and Astrophysical Fluid Dynamics, 1(1-2), 249-272. https://doi.org/10.1080/03091927009365774

Gill, A. E. (1982). Atmosphere-ocean dynamics, International Geophysics Series (Vol. 30, p. 666). San Diego, CA: Academic Press.

Ginis, I. (2002). Tropical cyclone-ocean interactions. Advances in Fluid Mechanics, 33, 83-114.

Ginis, I., \& Sutyrin, G. (1995). Hurricane-generated depth-averaged currents and sea surface elevation. Journal of Physical Oceanography, 25(6), 1218-1242. https://doi.org/10.1175/1520-0485(1995)025<1218:HGDACA > 2.0.CO;2

Holland, G. J. (1980). An analytical model of the wind and pressure profiles in hurricanes. Monthly Weather Review, 108(8), $1212-1218$. https://doi.org/10.1175/1520-0493(1980)108<1212:AAMOTW>2.0.CO;2

Huang, S. M., \& Oey, L. Y. (2015). Right-side cooling and phytoplankton bloom in the wake of a tropical cyclone. Journal of Geophysical Research: Oceans, 120, 5735-5748. https://doi.org/10.1002/2015JC010896

Kolodziejczyk, N., Prigent-Mazella, A., \& Gaillard, F. (2017). ISAS-15 temperature and salinity gridded fields. SEANOE. http://doi.org/ $10.17882 / 52367$

Kudryavtsev, V., Dulov, V., Shrira, V., \& Malinovsky, V. (2008). On vertical structure of wind-driven sea surface currents. Journal of Physical Oceanography, 38(10), 2121-2144. https://doi.org/10.1175/2008JPO3883.1

Kudryavtsev, V., Golubkin, P., \& Chapron, B. (2015). A simplified wave enhancement criterion for moving extreme events. Journal of Geophysical Research: Oceans, 120, 7538-7558. https://doi.org/10.1002/2015JC011284

Kudryavtsev, V., Monzikova, A., Combot, C., Chapron, B., Reul, N., \& Quilfen, Y. (2019). A simplified model for the baroclinic and barotropic ocean response to moving tropical cyclones: 1. Satellite Observations. Journal of Geophysical Research: Oceans, 124. https://doi. org/10.1029/2018JC014746

Lighthill, M. J. (1967). On waves generated in dispersive systems by travelling forcing effects with applications to the dynamics of rotating fluids. Journal of Fluid Mechanics, 27(4), 725-752. https://doi.org/10.1017/S0022112067002563

Lloyd, I. D., \& Vecchi, G. A. (2011). Observational evidence for oceanic controls on hurricane intensity. Journal of Climate, 24(4), 1138-1153. https://doi.org/10.1175/2010JCLI3763.1

Niiler, P. P. (1975). Deepening of the wind-mixed layer. Journal of Marine Research, 33(3), 405-421.

Orlanski, I., \& Polinsky, L. J. (1983). Ocean response to mesoscale atmospheric forcing. Tellus, 35A(4), 296-323. https://doi.org/10.1111/ j.1600-0870.1983.tb00205.x

Pollard, R. T., Rhines, P. B., \& Thompson, R. O. (1972). The deepening of the wind-mixed layer. Geophysical and Astrophysical Fluid Dynamics, 4(1), 381-404. https://doi.org/10.1080/03091927208236105

Powell, M. D., Vickery, P. J., \& Reinhold, T. A. (2003). Reduced drag coefficient for high wind speeds in tropical cyclones. Nature, 422(6929), 279-283. https://doi.org/10.1038/nature01481

Price, J. F. (1981). Upper ocean response to a hurricane. Journal of Physical Oceanography, 11(2), 153-175. https://doi.org/10.1175/15200485(1981)011<0153:UORTAH > 2.0.CO;2 
Price, J. F., Sanford, T. B., \& Forristall, G. Z. (1994). Forced stage response to a moving hurricane. Journal of Physical Oceanography, 24(2), 233-260. https://doi.org/10.1175/1520-0485(1994)024<0233:FSRTAM >2.0.CO;2

Price, J. F., Weller, R. A., \& Pinkel, R. (1986). Diurnal cycling: Observations and models of the upper ocean response to diurnal heating, cooling, and wind mixing. Journal of Geophysical Research, 91(C7), 8411-8427. https://doi.org/10.1029/JC091iC07p08411

Reichl, B. G., Ginis, I., Hara, T., Thomas, B., Kukulka, T., \& Wang, D. (2016). Impact of sea-state-dependent Langmuir turbulence on the ocean response to a tropical cyclone. Monthly Weather Review, 144(12), 4569-4590. https://doi.org/10.1175/MWR-D-16-0074.1

Reul, N., Chapron, B., Lee, T., Donlon, C., Boutin, J., \& Alory, G. (2014). Sea surface salinity structure of the meandering Gulf Stream revealed by SMOS sensor. Geophysical Research Letters, 119, 8271-8295. https://doi.org/10.1002/2014JC010107

Reul, N., Chapron, B., Zabolotskikh, E., Donlon, C., Mouche, A., Tenerelli, J., et al. (2017). A new generation of tropical cyclone size measurements from space. Bulletin of the American Meteorological Society, 98(11), 2367-2385. https://doi.org/10.1175/BAMS-D-1500291.1

Sanford, T. B., Price, J. F., Girton, J. B., \& Webb, D. C. (2007). Highly resolved observations and simulations of the ocean response to a hurricane. Geophysical Research Letters, 34, L13604. https://doi.org/10.1029/2007GL029679

Schade, L. R. (2000). Tropical cyclone intensity and sea surface temperature. Journal of the Atmospheric Sciences, 57(18), 3122-3130. https:// doi.org/10.1175/1520-0469(2000)057<3122:TCIASS >2.0.CO;2

Shea, D. J., \& Gray, W. M. (1973). The hurricane's inner core region. I. Symmetric and asymmetric structure. Journal of the Atmospheric Sciences, 30(8), 1544-1564. https://doi.org/10.1175/1520-0469(1973)030<1544:THICRI >2.0.CO;2

Skyllingstad, E. D., Smyth, W. D., \& Crawford, G. B. (2000). Resonant wind-driven mixing in the ocean boundary layer. Journal of Physical Oceanography, 30(8), 1866-1890. https://doi.org/10.1175/1520-0485(2000)030<1866:RWDMIT>2.0.CO;2

Sraj, I., Iskandarani, M., Srinivasan, A., Thacker, W. C., Winokur, J., Alexanderian, A., \& Knio, O. M. (2013). Bayesian inference of drag parameters using AXBT data from Typhoon Fanapi. Monthly Weather Review, 141(7), 2347-2367. https://doi.org/10.1175/MWR-D-1200228.1

Stoney, L., Walsh, K., Babanin, A. V., Ghantous, M., Govekar, P., \& Young, I. (2017). Simulated ocean response to tropical cyclones: The effect of a novel parameterization of mixing from unbroken surface waves. Journal of Advances in Modeling Earth Systems, 9, 759-780. https://doi.org/10.1002/2016MS000878

Yablonsky, R. M., \& Ginis, I. (2009). Limitation of one-dimensional ocean models for coupled hurricane-ocean model forecasts. Monthly Weather Review, 137(12), 4410-4419. https://doi.org/10.1175/2009MWR2863.1

Yablonsky, R. M., \& Ginis, I. (2013). Impact of a warm ocean eddy's circulation on hurricane induced sea surface cooling with implications for hurricane intensity. Monthly Weather Review, 141(3), 997-1021. https://doi.org/10.1175/MWR-D-12-00248.1

Yablonsky, R. M., Ginis, I., \& Thomas, B. (2015). Ocean modeling with flexible initialization for improved coupled tropical cyclone-ocean model prediction. Environmental Modelling \& Software, 67, 26-30. https://doi.org/10.1016/j.envsoft.2015.01.003

Young, I. R. (2006). Directional spectra of hurricane wind waves. Journal of Geophysical Research, 111, C08020. https://doi.org/10.1029/ 2006JC003540

Zedler, S. E., Kanschat, G., Hoteit, I., \& Korty, R. (2013). Estimation of the drag coefficient from the upper ocean response to a hurricane: A variational data assimilation approach. Ocean Modelling, 68(2013), 57-71. https://doi.org/10.1016/j.ocemod.2013.04.004

Zedler, S. E., Kanschat, G., Korty, R., \& Hoteit, I. (2012). A new approach for the determination of the drag coefficient from the upper ocean response to a tropical cyclone: A feasibility study. Journal of Oceanography, 68(2), 227-241. https://doi.org/10.1007/s10872-011-0092-6

Zedler, S. E., Niiler, P. P., Stammer, D., Terrill, E., \& Morzel, J. (2009). Ocean's response to Hurricane Frances and its implications for drag coefficient parameterization at high wind speeds. Journal of Geophysical Research, 114, C04016. https://doi.org/10.1029/2008JC005205

Zilitinkevich, S. S., Chalikov, D. V., \& Resnyansky, Y. D. (1979). Modelling the oceanic upper layer. Oceanologica Acta, 2(2), $219-240$. 